\title{
Finite type invariants of rational homology 3-spheres
}

\author{
DELPHine Moussard
}

\begin{abstract}
We consider the rational vector space generated by all rational homology spheres up to orientation-preserving homeomorphism, and the filtration defined on this space by Lagrangian-preserving rational homology handlebody replacements. We identify the graded space associated with this filtration with a graded space of augmented Jacobi diagrams.
\end{abstract}

57M27; 57N10, 57N65

\section{Introduction}

\subsection{Finite type invariants}

The greatest achievements in the theories of finite type invariants are theorems that express the graded spaces associated with topological filtrations of vector spaces generated by knots or manifolds as combinatorial vector spaces generated by Feynman diagrams. The two main examples of these theorems, that are useful to classify invariants and to evaluate their power, concern the Vassiliev filtration of the space generated by the knots in $S^{3}$, and the Goussarov-Habiro filtration of the space generated by the integral homology 3-spheres ( $\mathbb{Z H S}$ ), that are oriented compact 3-manifolds with the same integral homology as $S^{3}$. The graded space associated with the Vassiliev filtration was identified with a space of Jacobi diagrams by an isomorphism induced by the Kontsevich integral [6] (see Bar-Natan [2]). Several filtrations of the space generated by the $\mathbb{Z}$ HSs were defined. In [3], Garoufalidis, Goussarov and Polyak compared various filtrations, and defined a surjective map from a graded space of Jacobi diagrams to the graded space associated with the Goussarov-Habiro filtration. In [8], Le proved that this map is an isomorphism by showing that the LMO invariant that he constructed in [9] with the help of Murakami and Ohtsuki is a universal finite type invariant of $\mathbb{Z}$ HSs. In [1], Auclair and Lescop defined the Goussarov-Habiro filtration and the properties of the graded space, algebraically, using Lagrangian-preserving integral homology handlebody replacements.

In this article, we will consider the rational vector space generated by all the rational homology spheres $(\mathbb{Q} H S)$, that are the oriented compact 3-manifolds with the same 
rational homology as $S^{3}$. We will define a filtration on this space by means of LPsurgeries, that are Lagrangian-preserving rational homology handlebody replacements. Our main result (Theorem 1.7) identifies the graded space associated with this filtration with a graded space of diagrams. The role of the LMO invariant in the integral case will be held here by the KKT invariant of rational homology spheres constructed by Kontsevich, and proved to be a universal finite type invariant of $\mathbb{Z} H S$ s by Kuperberg and Thurston in [7]. Lescop has proved in [10] that the KKT invariant $Z_{K K T}=\left(Z_{n, K K T}\right)_{n \in \mathbb{N}}$ satisfies a universality property with respect to LP-surgeries. Massuyeau has proved in [11] that the LMO invariant $Z_{L M O}=\left(Z_{n, L M O}\right)_{n \in \mathbb{N}}$ satisfies the same property. As we prove at the end of Section 6, these results and our main theorem imply that $Z_{L M O}$ and $Z_{K K T}$ are equivalent in the following sense.

Theorem 1.1 Let $M$ and $N$ be $\mathbb{Q H S s}$ such that $\left|H_{1}(M ; \mathbb{Z})\right|=\left|H_{1}(N ; \mathbb{Z})\right|$, where $|\cdot|$ denotes the cardinality. Then, for any $n \in \mathbb{N}$,

$\left(Z_{k, L M O}(M)=Z_{k, L M O}(N)\right.$ for all $\left.k \leq n\right)$

$$
\Leftrightarrow\left(Z_{k, K K T}(M)=Z_{k, K K T}(N) \text { for all } k \leq n\right) .
$$

\subsection{The Goussarov-Habiro filtration}

Throughout the article, the manifolds will be compact, connected and oriented. When it does not seem to cause confusion, we will use the same notation for a curve and its homology class.

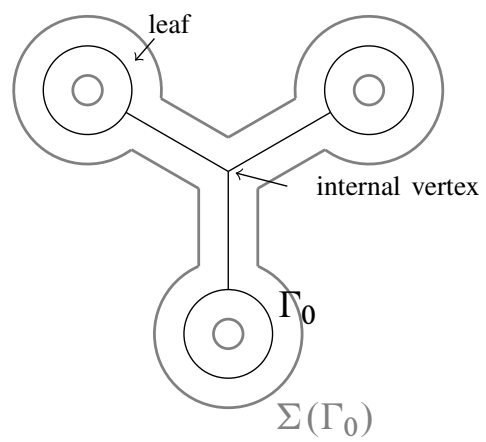

Figure 1: The standard Y-graph

The standard $Y$-graph is the graph $\Gamma_{0} \subset \mathbb{R}^{2}$ represented in Figure 1. With $\Gamma_{0}$ is associated a regular neighborhood $\Sigma\left(\Gamma_{0}\right)$ of $\Gamma_{0}$ in the plane.

Consider a 3-manifold $M$ and an embedding $h: \Sigma\left(\Gamma_{0}\right) \rightarrow M$. The image $\Gamma$ of $\Gamma_{0}$ is a $Y$-graph, and $\Sigma(\Gamma)=h\left(\Sigma\left(\Gamma_{0}\right)\right)$ is the associated surface of $\Gamma$. The Y-graph $\Gamma$ 
is equipped with the framing induced by $\Sigma(\Gamma)$. The looped edges of a Y-graph are called leaves. The vertex incident to three different edges is the internal vertex.
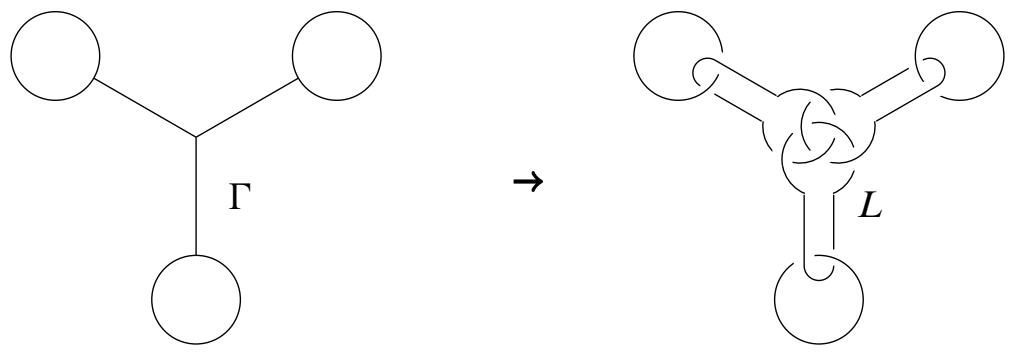

Figure 2: Y-graph and associated surgery link

Consider a Y-graph $\Gamma$ in a 3-manifold $M$. Associate with $\Gamma$ the six-component link $L$ represented in Figure 2. The Borromean surgery on $\Gamma$ is the surgery along the framed link $L$. As proved by Matveev in [12], a Borromean surgery can be realized by cutting a genus 3 handlebody (a regular neighborhood of the Y-graph) and regluing it another way. A $Y$-link in a 3-manifold is a collection of disjoint Y-graphs.

Consider the rational vector space $\mathcal{F}_{0}^{\mathbb{Z}}$ generated by all $\mathbb{Z}$ HSs up to orientationpreserving homeomorphism. Let $\mathcal{F}_{n}^{\mathbb{Z}}$ denote the subspace generated by all the

$$
[M ; \Gamma]=\sum_{I \subset\{1, \ldots, n\}}(-1)^{|I|} M\left(\bigcup_{i \in I} \Gamma_{i}\right),
$$

where $M$ is a $\mathbb{Z H S}, \Gamma_{i}$ are disjoint Y-graphs in $M, \Gamma=\bigcup_{i=1}^{n} \Gamma_{i}$, and $M\left(\bigcup_{i \in I} \Gamma_{i}\right)$ is the manifold obtained from $M$ by surgery on the $\Gamma_{i}$ for $i \in I$. Here and in all the article, $|I|$ stands for the cardinality of the set $I$. The associated quotients $\mathcal{G}_{n}^{\mathbb{Z}}=\mathcal{F}_{n}^{\mathbb{Z}} / \mathcal{F}_{n+1}^{\mathbb{Z}}$ can be described in terms of Jacobi diagrams.
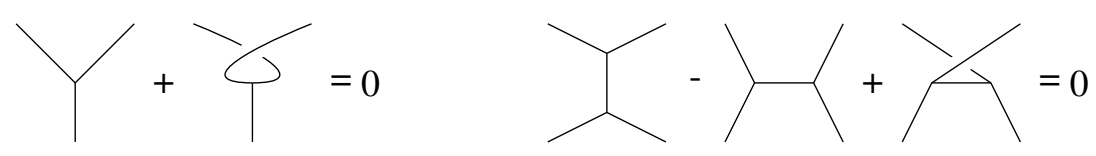

Figure 3: AS and IHX relations

A Jacobi diagram is a trivalent graph with oriented vertices. An orientation of a vertex of such a diagram is a cyclic order of the three half-edges that meet at this vertex. In the pictures, this orientation is induced by the cyclic order diagram is half the number of its vertices. Note that it is an integer. Let $\mathcal{A}_{n}$ denote the 
rational vector space generated by all degree $n$ Jacobi diagrams, quotiented out by the AS and IHX relations (Figure 3). The space $\mathcal{A}_{0}$ is generated by the empty diagram. Let $\mathcal{A}_{n}^{c}$ denote the subspace of $\mathcal{A}_{n}$ generated by the connected diagrams.

Let $\Gamma$ be a Jacobi diagram of degree $n$. Let $\varphi: \Gamma \hookrightarrow \mathbb{R}^{3}$ be an embedding such that the orthogonal projection on $\mathbb{R}^{2} \times\{0\}$ of $\varphi(\Gamma)$ is regular, and hence induces a framing of $\varphi(\Gamma)$. Now associate a Y-link $\widetilde{\Gamma}$ in $S^{3}$ with $\Gamma$ by replacing all edges of $\varphi(\Gamma)$ as indicated in Figure 4.

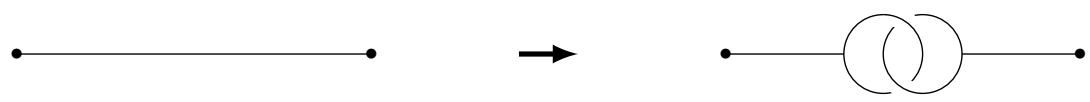

Figure 4: Replacement of an edge
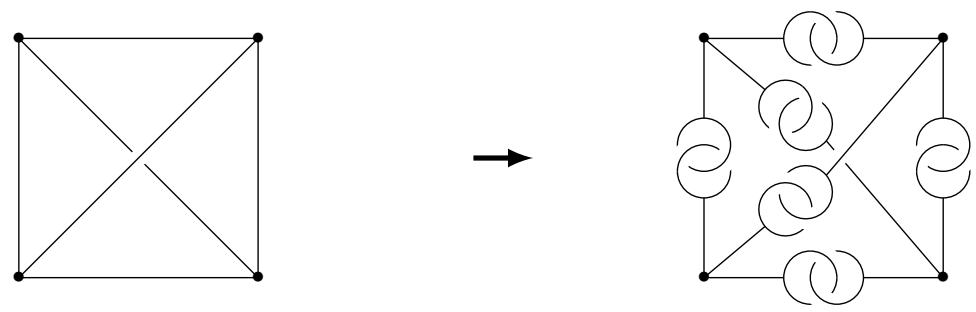

Figure 5: Jacobi diagram and associated Y-link

Lemma 1.2 (Garoufalidis, Goussarov and Polyak [3, Corollaries 4.2, 4.6, Theorem 4.11]) The bracket $\left[S^{3} ; \widetilde{\Gamma}\right] \in \mathcal{G}_{2 n}^{\mathbb{Z}}$ only depends on the class of $\Gamma$ in $\mathcal{A}_{n}$. Hence it defines

$$
\begin{aligned}
\Phi: \mathcal{A}_{n} & \rightarrow \mathcal{G}_{2 n}^{\mathbb{Z}}, \\
\Gamma & \mapsto\left[S^{3} ; \Gamma\right]:=\left[S^{3} ; \widetilde{\Gamma}\right] .
\end{aligned}
$$

Theorem 1.3 (Garoufalidis, Goussarov and Polyak [3], Habiro [4], Le [8]) For $n$ odd, $\mathcal{G}_{n}^{\mathbb{Z}}=0$. For $n$ even, the map $\Phi: \mathcal{A}_{\frac{n}{2}} \rightarrow \mathcal{G}_{n}^{\mathbb{Z}}$ is an isomorphism.

\subsection{Statement of the results}

We first define the filtration on the rational vector space $\mathcal{F}_{0}$ generated by all $\mathbb{Q} H S s$ up to orientation-preserving homeomorphism.

Definition 1.4 For $g \in \mathbb{N}$, a genus $g$ rational (respectively integral) homology handlebody $(\mathbb{Q H H}$, respectively $\mathbb{Z} H \mathrm{H})$ is a 3-manifold which is compact, oriented, and 
which has the same homology with rational (respectively integral) coefficients as the standard genus $g$ handlebody.

Such a $\mathbb{Q H H}$ (respectively $\mathbb{Z} H H$ ) is connected, and its boundary is necessarily homeomorphic to the standard genus $g$ surface.

Definition 1.5 The Lagrangian $\mathcal{L}_{A}$ of a $\mathbb{Q H H} A$ is the kernel of the map

$$
i_{*}: H_{1}(\partial A ; \mathbb{Q}) \rightarrow H_{1}(A ; \mathbb{Q})
$$

induced by the inclusion. Two $\mathbb{Q H H s} A$ and $B$ have $L P$-identified boundaries if we have a homeomorphism $h: \partial A \rightarrow \partial B$ such that $h_{*}\left(\mathcal{L}_{A}\right)=\mathcal{L}_{B}$.

The Lagrangian of a $\mathbb{Q H H} A$ is indeed a Lagrangian subspace of $H_{1}(\partial A ; \mathbb{Q})$ with respect to the intersection form.

Consider a $\mathbb{Q} H S M$, a $\mathbb{Q H H} A \subset M$, and a $\mathbb{Q H H} B$ whose boundary is LP-identified with $\partial A$. Set $M(B / A)=(M \backslash \operatorname{Int} A) \cup_{\partial A=\partial B} B$. We say that the $\mathbb{Q H S} M(B / A)$ is obtained from $M$ by Lagrangian preserving surgery, or LP-surgery. Note that a Borromean surgery is a special type of LP-surgery. If $\left(A_{i}\right)_{1 \leq i \leq n}$ is a family of disjoint $\mathbb{Q} H H s$ in $M$, and if, for each $i, B_{i}$ is a $\mathbb{Q H H}$ whose boundary is LP-identified with $\partial A_{i}$, we denote by $M\left(\left(B_{i} / A_{i}\right)_{1 \leq i \leq n}\right)$ the manifold obtained from $M$ by the $n$ LP-surgeries $\left(B_{i} / A_{i}\right)$.

Let $\mathcal{F}_{n}$ denote the subspace of $\mathcal{F}_{0}$ generated by the

$$
\left[M ;\left(\frac{B_{i}}{A_{i}}\right)_{1 \leq i \leq n}\right]=\sum_{I \subset\{1, \ldots, n\}}(-1)^{|I|} M\left(\left(\frac{B_{i}}{A_{i}}\right)_{i \in I}\right)
$$

for all $\mathbb{Q}$ HSs $M$ and all families of $\mathbb{Q H H s}\left(A_{i}, B_{i}\right)_{1 \leq i \leq n}$, where the $A_{i}$ are embedded in $M$ and disjoint, and each $\partial B_{i}$ is LP-identified with the corresponding $\partial A_{i}$. Since $\mathcal{F}_{n+1} \subset \mathcal{F}_{n}$, this defines a filtration. Set $\mathcal{G}_{n}=\mathcal{F}_{n} / \mathcal{F}_{n+1}$ and $\mathcal{G}=\bigoplus_{n \in \mathbb{N}} \mathcal{G}_{n}$.

Definition 1.6 A finite type invariant of degree at most $n$ of rational homology spheres is a linear map $\lambda: \mathcal{F}_{0} \rightarrow \mathbb{Q}$ such that $\lambda\left(\mathcal{F}_{n+1}\right)=0$. It is said to be additive if $\lambda(M \sharp N)=\lambda(M)+\lambda(N)$ for all $\mathbb{Q H S s} M$ and $N$.

Let $\mathcal{I}_{n}$ (respectively $\mathcal{I}_{n}^{c}$ ) denote the rational vector space of all invariants (respectively additive invariants) of degree at most $n$. Set $\mathcal{H}_{n}=\mathcal{I}_{n} / \mathcal{I}_{n-1}$ and $\mathcal{H}=\bigoplus_{n \in \mathbb{N}} \mathcal{H}_{n}$. Note that $\mathcal{I}_{n}$ is canonically isomorphic to $\left(\mathcal{F}_{0} / \mathcal{F}_{n+1}\right)^{*}:=\operatorname{Hom}\left(\mathcal{F}_{0} / \mathcal{F}_{n+1}, \mathbb{Q}\right)$. We have an exact sequence

$$
0 \rightarrow \mathcal{G}_{n} \rightarrow \frac{\mathcal{F}_{0}}{\mathcal{F}_{n+1}} \rightarrow \frac{\mathcal{F}_{0}}{\mathcal{F}_{n}} \rightarrow 0
$$


Since the functor $\operatorname{Hom}(\cdot, \mathbb{Q})$ is exact, the dual sequence

$$
0 \rightarrow \mathcal{I}_{n-1} \rightarrow \mathcal{I}_{n} \rightarrow\left(\mathcal{G}_{n}\right)^{*} \rightarrow 0
$$

is also exact. Thus $\mathcal{H}_{n} \cong\left(\mathcal{G}_{n}\right)^{*}$.

We will call augmented diagram of degree $n$ the union of a Jacobi diagram of degree $k \leq \frac{n}{2}$ and of $(n-2 k)$ weighted vertices, where the weights are prime integers.

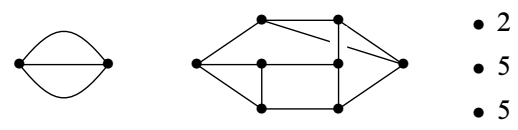

Figure 6: Augmented diagram of degree 13

Note that the degree of an augmented diagram is equal to its number of vertices. Let $\mathcal{A}_{n}^{\text {aug }}$ denote the rational vector space generated by all augmented diagrams of degree $n$, quotiented out by the AS and IHX relations. The main goal of this article is to prove the following theorem.

Theorem 1.7 For $n \in \mathbb{N}, \mathcal{A}_{n}^{\text {aug }} \cong \mathcal{G}_{n}$.

This result will follow from Propositions 1.8, 1.11 and 6.9. An isomorphism can be described in the following way. Consider an augmented diagram $\Gamma_{a}$ of degree $n$ given by a Jacobi diagram $\Gamma$ of degree $k$, and $(n-2 k)$ vertices with weights $\left(p_{i}\right)_{1 \leq i \leq n-2 k}$. Define $\varphi(\Gamma) \subset S^{3}$ and the associated Y-link $\widetilde{\Gamma}$ as before. For each $i$, consider a rational homology ball $B_{p_{i}}$ such that $H_{1}\left(B_{p_{i}} ; \mathbb{Z}\right)=\mathbb{Z} / p_{i} \mathbb{Z}$. Then define the image of $\Gamma_{a}$ as $\left[S^{3} ; \tilde{\Gamma},\left(B_{p_{i}} / B^{3}\right)_{1 \leq i \leq n-2 k}\right] \in \mathcal{G}_{n}$.

Since connected sums are LP-surgeries of genus 0 , one can easily see that $\mathcal{G}_{0} \cong \mathbb{Q} S^{3}$. In Section 4, we give a description of $\mathcal{G}_{1}$.

Proposition 1.8 For any prime integer $p$, fix a $\mathbb{Q H S} M_{p}$ such that $\left|H_{1}\left(M_{p}\right)\right|=p$. Then $\left(M_{p}-S^{3}\right)_{p}$ prime is a basis for $\mathcal{G}_{1}$.

Remark The $\mathbb{Q}$ HSs $M_{p}$ are not unique in $\mathcal{F}_{0}$, but we will see in Section 4.1 that they are unique modulo $\mathcal{F}_{2}$.

We will show in Section 4.1 that the family $\left(M_{p}-S^{3}\right)_{p}$ prime generates $\mathcal{G}_{1}$. To see that it is a basis, we will prove the following proposition in Section 4.2.

For a prime integer $p$, let $v_{p}$ denote the $p$-adic valuation, defined on $\mathbb{N} \backslash\{0\}$ by $v_{p}\left(p^{k} n\right)=k$ if $n$ is prime to $p$. 
Proposition 1.9 For any prime integer $p$, define a linear map $v_{p}$ on $\mathcal{F}_{0}$ by setting $v_{p}(M)=v_{p}\left(\left|H_{1}(M)\right|\right)$ when $M$ is a $\mathbb{Q H S}$. Then $v_{p}$ is a degree 1 invariant of $\mathbb{Q H S s}$.

Since $v_{p}\left(M_{p}\right)=1, v_{p}\left(M_{q}\right)=0$ for any prime $q \neq p$, and $v_{p}\left(S^{3}\right)=0$ for any prime $p$, this result shows that the family $\left(M_{p}-S^{3}\right)_{p}$ prime is free.

Corollary 1.10 $\mathcal{I}_{1} / \mathcal{I}_{0}=\mathcal{I}_{1}^{c}=\prod_{p \text { prime }} \mathbb{Q} v_{p}$.

In Section 5, we prove the following.

Proposition 1.11 For $n>1, \mathcal{I}_{n}^{c} / \mathcal{I}_{n-1}^{c} \cong\left(\mathcal{A}_{n / 2}^{c}\right)^{*}$ if $n$ is even, and $\mathcal{I}_{n}^{c} / \mathcal{I}_{n-1}^{c} \cong 0$ if $n$ is odd.

In this proof, we will use the description of finite type invariants of degree 1 of framed rational homology tori given in Section 5.1.

In Section 6, we use the structures of graded algebras on $\mathcal{G}$ and $\mathcal{H}$ in order to show that any finite type invariant $\lambda$ such that $\lambda\left(S^{3}\right)=0$ can be written as a sum of products of additive invariants. More precisely, let $\mathcal{I}_{n}^{\pi}$ denote the subspace of $\mathcal{I}_{n}$ generated by all the products $\prod_{1 \leq i \leq k} \lambda_{i}$, where $k>1$, the $\lambda_{i}$ are additive invariants of degree $k_{i}<n$, and $\sum_{1 \leq i \leq k} k_{i} \leq n$. Our version of the Milnor-Moore theorem about the structure of Hopf algebras implies the following.

Proposition 1.12 For all $n>0, \mathcal{I}_{n}=\mathcal{I}_{0} \oplus \mathcal{I}_{n}^{c} \oplus \mathcal{I}_{n}^{\pi}$.

We will obtain this result as a consequence of Proposition 6.9.

In order to describe the spaces of additive invariants, we shall prove that LP-surgeries can be reduced to more specific moves.

Definition 1.13 Consider a positive integer $d$. We call $d$-torus a rational homology torus such that:

- $H_{1}\left(\partial T_{d} ; \mathbb{Z}\right)=\mathbb{Z} \alpha \oplus \mathbb{Z} \beta$, with $\langle\alpha, \beta\rangle=1 ;$

- $d \alpha=0$ in $H_{1}\left(T_{d} ; \mathbb{Z}\right)$;

- $\beta=d \gamma$ in $H_{1}\left(T_{d} ; \mathbb{Z}\right)$, where $\gamma$ is a curve in $T_{d}$;

- $H_{1}\left(T_{d} ; \mathbb{Z}\right)=\mathbb{Z}_{d} \alpha \oplus \mathbb{Z} \gamma$.

Definition 1.14 An elementary surgery is an LP-surgery among the following ones:

(1) connected sum (genus 0);

(2) LP-replacement of a standard torus by a $d$-torus (genus 1 );

(3) Borromean surgery (genus 3). 
In Section 2, we prove the following.

Theorem 1.15 If $A$ and $B$ are two $\mathbb{Q H H s}$ with $L P$-identified boundaries, then $B$ can be obtained from $A$ by a finite sequence of elementary surgeries and their inverses in the interior of the $\mathbb{Q} H H s$.

This proposition generalizes a result of Auclair and Lescop [1, Lemma 4.11] which says that any two $\mathbb{Z H H s}$ with LP-identified boundaries can be obtained from one another by a finite sequence of Borromean surgeries in the interior of the $\mathbb{Z} H H s$.

In Section 3, we recall some facts about Borromean surgeries proved by Garoufalidis, Goussarov and Polyak in [3], and we give consequences of these facts that are useful in the sequel.

Acknowledgements I wish to thank the referee for his careful reading. My thanks also go to my advisor, Christine Lescop, for her helpful advice and rigorous supervision.

\section{Elementary surgeries}

\subsection{Homological properties of $\mathbb{Q} H H s$}

Definition 2.1 Consider the genus $g$ compact surface $\Sigma_{g}$. A basis $\left(\alpha_{i}, \beta_{i}\right)_{1 \leq i \leq g}$ of $H_{1}\left(\Sigma_{g} ; \mathbb{Z}\right)$ is called symplectic if the matrix in $\left(\alpha_{1}, \ldots, \alpha_{g}, \beta_{1}, \ldots, \beta_{g}\right)$ of the intersection form is $\left(\begin{array}{cc}0 & I_{g} \\ -I_{g} & 0\end{array}\right)$.

Notation We denote by $\operatorname{Tors}(H)$ the torsion submodule of a module $H$.

Lemma 2.2 If $A$ is a genus $g \mathbb{Q H H}$, then

- $H_{1}(A ; \mathbb{Z}) \cong \mathbb{Z}^{g} \oplus \operatorname{Tors}\left(H_{1}(A ; \mathbb{Z})\right) ;$

- $H_{2}(A ; \mathbb{Z})=0$;

- $H_{2}(A, \partial A ; \mathbb{Z}) \cong\left(H_{1}(A ; \mathbb{Z}) / \operatorname{Tors}\left(H_{1}(A ; \mathbb{Z})\right)\right)^{*} \cong \mathbb{Z}^{g}$.

Proof The first point is given by $H_{1}(A ; \mathbb{Z}) \otimes \mathbb{Q} \cong H_{1}(A ; \mathbb{Q}) \cong \mathbb{Q}^{g}$.

By the Poincaré duality, we have $H_{2}(A ; \mathbb{Z}) \cong H^{1}(A, \partial A ; \mathbb{Z})$. The universal coefficient theorem gives $H^{1}(A, \partial A ; \mathbb{Z}) \cong \operatorname{Hom}\left(H_{1}(A, \partial A ; \mathbb{Z}), \mathbb{Z}\right)$. Hence $H_{2}(A ; \mathbb{Z})$ is torsion free. Since $H_{2}(A ; \mathbb{Q})=0$, we get the second point.

The last point also follows from the Poincaré duality and the universal coefficient theorem:

$$
H_{2}(A, \partial A ; \mathbb{Z}) \cong H^{1}(A ; \mathbb{Z}) \cong \operatorname{Hom}\left(H_{1}(A ; \mathbb{Z}), \mathbb{Z}\right) \cong \mathbb{Z}^{g}
$$


Lemma 2.3 Consider a genus $g \mathbb{Q H H} A$ and the map $i_{*}: H_{1}(\partial A ; \mathbb{Z}) \rightarrow H_{1}(A ; \mathbb{Z})$ induced by the inclusion. Set

$$
\mathcal{L}_{A}^{\mathbb{Z}}=\operatorname{Ker} i_{*}, \quad \mathcal{L}_{A}^{T}=\left(i_{*}\right)^{-1}\left(\operatorname{Tors}\left(H_{1}(A ; \mathbb{Z})\right)\right) .
$$

Then there is a symplectic basis $\left(\alpha_{i}, \beta_{i}\right)_{1 \leq i \leq g}$ of $H_{1}(\partial A ; \mathbb{Z})$, a family $\left(\gamma_{i}\right)_{1 \leq i \leq g}$ of curves in $A$, and positive integers $d_{i}, 1 \leq i \leq g$, such that

$$
\mathcal{L}_{A}^{\mathbb{Z}}=\bigoplus_{1 \leq i \leq g} \mathbb{Z}\left(d_{i} \alpha_{i}\right), \quad \mathcal{L}_{A}^{T}=\bigoplus_{1 \leq i \leq g} \mathbb{Z} \alpha_{i}, \quad \frac{H_{1}(A ; \mathbb{Z})}{\operatorname{Tors}\left(H_{1}(A ; \mathbb{Z})\right)}=\bigoplus_{1 \leq i \leq g} \mathbb{Z} \gamma_{i},
$$

and $\beta_{i}=d_{i} \gamma_{i}$ in $H_{1}(A ; \mathbb{Z}) / \operatorname{Tors}\left(H_{1}(A ; \mathbb{Z})\right)$ for $1 \leq i \leq g$.

In particular,

$$
\frac{\mathcal{L}_{A}^{T}}{\mathcal{L}_{A}^{\mathbb{Z}}}, \quad \frac{H_{1}(A ; \mathbb{Z})}{\operatorname{Tors}\left(H_{1}(A ; \mathbb{Z})\right) \oplus\left(\bigoplus_{1 \leq i \leq g} \mathbb{Z} \beta_{i}\right)},
$$

are isomorphic to $\prod_{1 \leq i \leq g} \mathbb{Z} / d_{i} \mathbb{Z}$.

Proof The exact sequence over $\mathbb{Z}$ associated with $(A, \partial A)$ yields the exact sequence

$$
0 \rightarrow H_{2}(A, \partial A) \rightarrow H_{1}(\partial A) \stackrel{i_{*}}{\longrightarrow} H_{1}(A) .
$$

Thus $\mathcal{L}_{A}^{\mathbb{Z}}$ is a free submodule of rank $g$ of $H_{1}(\partial A ; \mathbb{Z})$. Hence there exist a basis $\left(\alpha_{i}, \beta_{i}\right)_{1 \leq i \leq g}$ of $H_{1}(\partial A ; \mathbb{Z})$, and integers $d_{i}>0,1 \leq i \leq g$, such that $\left(d_{i} \alpha_{i}\right)_{1 \leq i \leq g}$ is a basis of $\mathcal{L}_{A}^{\mathbb{Z}}$. It follows that $\mathcal{L}_{A}^{T}=\bigoplus_{1 \leq i \leq g} \mathbb{Z} \alpha_{i}$. Since the intersection form is trivial on $\mathcal{L}_{A}^{T}$, we can choose the $\beta_{i}$ in such a way that the basis $\left(\alpha_{i}, \beta_{i}\right)_{1 \leq i \leq g}$ is symplectic.

The boundary map $H_{2}(A, \partial A) \rightarrow H_{1}(\partial A)$ in the above exact sequence induces an isomorphism $H_{2}(A, \partial A ; \mathbb{Z}) \cong \mathcal{L}_{A}^{\mathbb{Z}}$. Thus we can choose a basis $\left(S_{i}\right)_{1 \leq i \leq g}$ of $H_{2}(A, \partial A ; \mathbb{Z})$ such that $\partial S_{i}=d_{i} \alpha_{i}$ for $1 \leq i \leq g$. Let $\left(\gamma_{i}\right)_{1 \leq i \leq g}$ denote the basis of $H_{1}(A ; \mathbb{Z}) / \operatorname{Tors}\left(H_{1}(A ; \mathbb{Z})\right)$ Poincaré dual to $\left(S_{i}\right)_{1 \leq i \leq g}$.

For $1 \leq i, j \leq g,\left\langle S_{j}, \beta_{i}\right\rangle_{A}=\left\langle d_{j} \alpha_{j}, \beta_{i}\right\rangle_{\partial A}=\delta_{i j} d_{i}$, where $\delta_{i j}$ is the Kronecker delta, equal to 1 if $i=j$ and 0 otherwise. Thus $\beta_{i}=d_{i} \gamma_{i}$ in $H_{1}(A ; \mathbb{Z}) / \operatorname{Tors}\left(H_{1}(A ; \mathbb{Z})\right)$.

Corollary 2.4 Let $A$ be a $\mathbb{Q H H}$. If the map $H_{1}(\partial A ; \mathbb{Z}) \rightarrow H_{1}(A ; \mathbb{Z})$, induced by the inclusion $\partial A \hookrightarrow A$, is surjective, then $A$ is a $\mathbb{Z H H}$. 




Figure 7: The handlebody $A$

\section{$2.2 d$-tori}

Lemma 2.5 For any positive integer $d$, there exists a $d$-torus $T_{d}$.

Proof Consider the standard genus 2 handlebody $A$ represented in Figure 7.

Consider a curve $c$ on $\partial A$ such that $c=a_{1}+d b_{2}$ in $H_{1}(\partial A ; \mathbb{Z})$. According to Meyerson in [13], since $c$ is primitive, it can be chosen simple and closed. The torus $T_{d}$ will be obtained from $A$ by adding a 2-handle to $A$ along $c$ as follows. Define $T_{d}=A \bigcup_{h}\left(D^{2} \times[-1,1]\right)$, where $h: \partial D^{2} \times[-1,1] \rightarrow \partial A$ is an embedding such that $h\left(\partial D^{2} \times\{0\}\right)=c$. We have $H_{1}\left(T_{d} ; \mathbb{Z}\right)=\left\langle b_{1}, b_{2} \mid d b_{2}=0\right\rangle$.

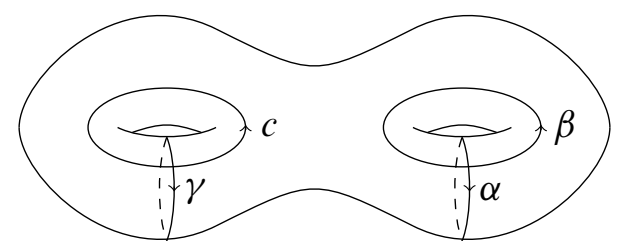

Figure 8: The surface $\partial A$

Moreover, we can define curves $\alpha, \beta, \gamma$, on $\partial A$, with $\alpha=b_{2}, \beta=-a_{2}-d b_{1}$ and $\gamma=-b_{1}$ in $H_{1}(\partial A ; \mathbb{Z})$ such that the boundary of $A$ is homeomorphic to the surface represented in Figure 8. Then we get that $H_{1}\left(T_{d} ; \mathbb{Z}\right)=\langle\gamma, \alpha \mid d \alpha=0\rangle$ and $H_{1}\left(\partial T_{d} ; \mathbb{Z}\right)=\mathbb{Z} \alpha \oplus \mathbb{Z} \beta$.

Given a curve $\gamma$ in a 3-manifold $M$, we will call exterior of $\gamma$ in $M$ the complement of the open tubular neighborhood of $\gamma$ in $M$.

Lemma 2.6 Let $d$ be a positive integer. Let $T_{d}$ be a $d$-torus. Let $\gamma$ be a curve in $T_{d}$ whose homology class generates $H_{1}\left(T_{d} ; \mathbb{Z}\right) / \operatorname{Tors}\left(H_{1}\left(T_{d} ; \mathbb{Z}\right)\right)$. Let $m(\gamma)$ and $\ell(\gamma)$ 
be respectively a meridian and a parallel of $\gamma$. For any integer $k$, there is a symplectic basis $(\alpha, \beta)$ of $H_{1}\left(\partial T_{d} ; \mathbb{Z}\right)$ such that $d \alpha=0$ in $H_{1}\left(T_{d} ; \mathbb{Z}\right)$, and such that the curve $\beta-d \ell(\gamma)+k m(\gamma)$ bounds a surface in the exterior of $\gamma$ in $T_{d}$.

Proof Let $X$ be the exterior of $\gamma$ in $T_{d}$. Consider a symplectic basis $\left(\alpha, \beta_{0}\right)$ of $H_{1}\left(\partial T_{d} ; \mathbb{Z}\right)$ such that $d \alpha=0$ and $\beta_{0}=d \gamma$ in $H_{1}\left(T_{d} ; \mathbb{Z}\right)$. There is an integer $k_{0}$ such that $\beta_{0}-d \ell(\gamma)+k_{0} m(\gamma)$ bounds a surface in $X$, ie, is trivial in $H_{1}(X ; \mathbb{Z})$. Since $d \alpha$ bounds a surface in $T_{d}$ that $\gamma$ meets once, $d \alpha-m(\gamma)$ is trivial in $H_{1}(X ; \mathbb{Z})$. Let $k$ be any integer, and set $\beta=\beta_{0}+\left(k_{0}-k\right) d \alpha$. The curve $\beta-d \ell(\gamma)+k m(\gamma)=$ $\left(\beta_{0}-d \ell(\gamma)+k_{0} m(\gamma)\right)+\left(k_{0}-k\right)(d \alpha-m(\gamma))$ is trivial in $H_{1}(X ; \mathbb{Z})$.

\subsection{Relating $\mathbb{Q} H H s$ by elementary surgeries}

In this subsection, we prove Theorem 1.15.

Definition 2.7 Consider a $\mathbb{Q} H H A$. Consider a simple closed curve $\gamma \subset A$. Consider a disk $D \subset \partial A$. Consider two distinct points $y$ and $z$ in Int $D$, and a path $s$ from $z$ to $y$ in Int $D$. Consider a cylinder $C=h\left(D^{2} \times[0,1]\right) \subset A$, where $h$ is an embedding such that:

- $h\left(D^{2} \times\{0\}\right)$ (respectively $\left.h\left(D^{2} \times\{1\}\right)\right)$ is a disk $D_{y}$ (respectively $\left.D_{z}\right)$ in Int $D$;

- $h(0,0)=y$ and $h(0,1)=z$;

- $C \cap \partial A=D_{y} \cup D_{z}$;

- $h(\{0\} \times[0,1]) \cup s$ is homologous to $\gamma$ in $A$.

We will call tunnel around $\gamma$ such a cylinder $C$.

Lemma 2.8 Let $A$ be a $\mathbb{Q H H}$ of genus $g$. Let $\gamma$ be a simple closed curve in $A$. Let $C$ be a tunnel around $\gamma$. Set $B=\overline{A \backslash C}$. Then $B$ is a $\mathbb{Q H H}$ of genus $g+1$.

Proof Consider the pair $(A, B)$. By excision, for $i \in \mathbb{N}$,

$$
H_{i}(A, B ; \mathbb{Q}) \cong H_{i}(C, C \cap B ; \mathbb{Q}) .
$$

Since $(C, C \cap B) \cong\left(D^{2} \times[0,1],\left(\partial D^{2}\right) \times[0,1]\right)$, it follows that $H_{i}(A, B ; \mathbb{Q})=0$ if $i \neq 2$, and $H_{2}(A, B ; \mathbb{Q}) \cong \mathbb{Q}$. The exact sequence over $\mathbb{Q}$ associated with the pair $(A, B)$ yields the exact sequence

$$
0 \rightarrow H_{2}(B) \rightarrow 0 \rightarrow H_{2}(A, B) \cong \mathbb{Q} \rightarrow H_{1}(B) \rightarrow H_{1}(A) \cong \mathbb{Q}^{g} \rightarrow 0 .
$$

Hence $H_{2}(B ; \mathbb{Q})=0$ and $H_{1}(B ; \mathbb{Q}) \cong \mathbb{Q}^{g+1}$. 
Lemma 2.9 Let $A$ be a $\mathbb{Q H H}$ of genus $g$. The quotient $H_{1}(A ; \mathbb{Z}) / H_{1}(\partial A ; \mathbb{Z})$ is a torsion module. Set $H_{1}(A ; \mathbb{Z}) / H_{1}(\partial A ; \mathbb{Z})=\bigoplus_{i=1}^{n} \mathbb{Z} / d_{i} \mathbb{Z} \mu_{i}$. Let $C_{i}, 1 \leq i \leq n$, be pairwise disjoint tunnels around the $\mu_{i}$. Then $B=\overline{A \backslash\left(\bigcup_{1 \leq i \leq n} C_{i}\right)}$ is a $\mathbb{Z H H}$ of genus $g+n$.

Proof The fact that $H_{1}(A ; \mathbb{Z}) / H_{1}(\partial A ; \mathbb{Z})$ is a torsion module follows from Lemma 2.3.

By Lemma 2.8, $B$ is a $\mathbb{Q H H}$ of genus $g+n$. Hence, by Corollary 2.4 , it suffices to show that the map $H_{1}(\partial B ; \mathbb{Z}) \rightarrow H_{1}(B ; \mathbb{Z})$ induced by the inclusion is surjective, or, equivalently, that $H_{1}(B, \partial B ; \mathbb{Z})$ is trivial. By excision, $H_{1}(B, \partial B ; \mathbb{Z})$ is isomorphic to $H_{1}\left(A, \partial A \cup\left(\bigcup_{1 \leq i \leq n} C_{i}\right) ; \mathbb{Z}\right)$, which is trivial by definition of the $C_{i}$ 's.

For a 3 -manifold $A$, let $l k_{A}$ : $\operatorname{Tors}\left(H_{1}(A ; \mathbb{Z})\right) \times \operatorname{Tors}\left(H_{1}(A ; \mathbb{Z})\right) \rightarrow \mathbb{Q} / \mathbb{Z}$ denote the linking form on $A$, defined in the following way. Consider disjoint representatives $\alpha, \beta$ of two homology classes in $\operatorname{Tors}\left(H_{1}(A ; \mathbb{Z})\right)$. Consider a surface $S \subset A$, transverse to $\beta$, such that $\partial S=k \alpha$ for some positive integer $k$. Then $l k_{A}(\alpha, \beta)=\frac{1}{k}\langle S, \beta\rangle$, where $\langle\cdot, \cdot\rangle$ is the algebraic intersection number in $A$. For a $\mathbb{Q H S} M$, the linking form $l k_{M}$ is defined on $H_{1}(M ; \mathbb{Z}) \times H_{1}(M ; \mathbb{Z})$, and it is known to be bilinear, symmetric and nondegenerate.

Lemma 2.10 Let $A$ be a $\mathbb{Q H H}$ of genus $g$. Assume $\mathcal{L}_{A}^{T} / \mathcal{L}_{A}^{\mathbb{Z}}=0$. Then there exists a $\mathbb{Q}$ HS $M$ such that $\left(H_{1}(M ; \mathbb{Z}), l k_{M}\right)$ is isomorphic to (Tors $\left.\left(H_{1}(A ; \mathbb{Z})\right), l k_{A}\right)$.

Proof By Lemma 2.3, there is a symplectic basis $\left(\alpha_{i}, \beta_{i}\right)_{1 \leq i \leq g}$ of $H_{1}(\partial A ; \mathbb{Z})$ such that the $\alpha_{i}$ are null-homologous in $A$, and $H_{1}(A ; \mathbb{Z})=\operatorname{Tors}\left(H_{1}(A)\right) \oplus\left(\bigoplus_{1 \leq i \leq g} \mathbb{Z} \beta_{i}\right)$. Consider a standard handlebody $H_{g}$, and a symplectic basis

$$
\left(a_{i}, b_{i}\right)_{1 \leq i \leq g} \text { of } H_{1}\left(\partial H_{g} ; \mathbb{Z}\right),
$$

where each $a_{i}$ bounds a disk in $H_{g}$. Construct a $\mathbb{Q H S} M$ by gluing $A$ and $H_{g}$ along their boundaries, in such a way that, for $1 \leq i \leq g, \alpha_{i}$ is identified with $b_{i}$, and $\beta_{i}$ is identified with $a_{i}$. We have $H_{1}(M ; \mathbb{Z}) \cong \operatorname{Tors}\left(H_{1}(A ; \mathbb{Z})\right)$. Moreover, the linkings of the curves in $A$ are preserved, thus the linking forms on $H_{1}(M)$ and $\operatorname{Tors}\left(H_{1}(A)\right)$ are isomorphic.

Lemma 2.11 Let $A$ and $A^{\prime}$ be $\mathbb{Q} H$ Hs of genus $g$ with LP-identified boundaries. Assume $\mathcal{L}_{A}^{T} / \mathcal{L}_{A}^{\mathbb{Z}}=0$ and $\mathcal{L}_{A^{\prime}}^{T} / \mathcal{L}_{A^{\prime}}^{\mathbb{Z}}=0$. If (Tors $\left.\left(H_{1}(A)\right), l k_{A}\right)$ is isomorphic to (Tors $\left.\left(H_{1}\left(A^{\prime}\right)\right), l k_{A^{\prime}}\right)$, then $A$ and $A^{\prime}$ can be obtained from one another by a finite sequence of Borromean surgeries. 
Proof Consider a basis $\left(\mu_{i}\right)_{1 \leq i \leq n}$ of Tors $\left(H_{1}(A)\right)$, and its image $\left(\mu_{i}^{\prime}\right)_{1 \leq i \leq n}$ under an isomorphism (Tors $\left.\left(H_{1}(A)\right), l k_{A}\right) \cong\left(\operatorname{Tors}\left(H_{1}\left(A^{\prime}\right)\right), l k_{A^{\prime}}\right)$. Fix framed representatives of the $\mu_{i}$ and $\mu_{i}^{\prime}$ such that $l k\left(\mu_{i}, \mu_{j}\right)=l k\left(\mu_{i}^{\prime}, \mu_{j}^{\prime}\right) \in \mathbb{Q}$ for $1 \leq i, j \leq n$. Consider pairwise disjoint tunnels $C_{i}$ (respectively $C_{i}^{\prime}$ ) around the $\mu_{i}$ (respectively $\mu_{i}^{\prime}$ ). Set $B=\overline{A \backslash\left(\bigcup_{1 \leq i \leq n} C_{i}\right)}$ and $B^{\prime}=\overline{A^{\prime} \backslash\left(\bigcup_{1 \leq i \leq n} C_{i}^{\prime}\right)}$. Extend the identification $\partial A \cong \partial A^{\prime}$ to an identification $\partial B \cong \partial B^{\prime}$ so that the longitude of each $\mu_{i}$ is identified with the longitude of the corresponding $\mu_{i}^{\prime}$. By Lemma $2.9, B$ and $B^{\prime}$ are $\mathbb{Z H H s}$ of genus $g+n$. The equality between the linking numbers ensures that the identification of their boundaries preserves the Lagrangian. Thus, by [1, Lemma 4.11], $B$ can be obtained from $B^{\prime}$ by a finite sequence of Borromean surgeries. Gluing back the cylinders, we get that $A$ can be obtained from $A^{\prime}$ by a finite sequence of Borromean surgeries.

Corollary 2.12 Consider a $\mathbb{Q H H} A$ such that $\mathcal{L}_{A}^{T} / \mathcal{L}_{A}^{\mathbb{Z}}=0$. Let $H_{g}$ be a standard handlebody such that $\partial H_{g}$ and $\partial A$ are LP-identified. Then there exists a $\mathbb{Q H S} M$ such that $A$ is obtained from $H_{g} \sharp M$ by a finite sequence of Borromean surgeries.

Lemma 2.13 Let $A$ be a genus $g \mathbb{Q H H}$. Let $H_{g}$ be a standard handlebody such that $\partial H_{g}$ and $\partial A$ are $L P$-identified. Assume there are a symplectic basis $\left(\alpha_{i}, \beta_{i}\right)_{1 \leq i \leq g}$ of $H_{1}(\partial A ; \mathbb{Z})$, a curve $\gamma$ in $A$, and a positive integer $d$ such that

$$
H_{1}(A ; \mathbb{Z})=\frac{\mathbb{Z}}{d \mathbb{Z}} \alpha_{1} \oplus \mathbb{Z} \gamma \oplus\left(\bigoplus_{2 \leq i \leq g} \mathbb{Z} \beta_{i}\right)
$$

and $\beta_{1}=d \gamma$. Then there are a solid torus $T_{0}$ embedded in $H_{g}$, a $d$-torus $T_{d}$, and an $L P$-identification $\partial T_{d} \cong \partial T_{0}$, such that $A$ is obtained from $H_{g}\left(T_{d} / T_{0}\right)$ by a finite sequence of Borromean surgeries.

Proof Consider a tunnel $C$ around $\gamma$ in $A$. Set $B=\overline{A \backslash C}$. By Lemma 2.9, $B$ is a $\mathbb{Z H H}$ of genus $g+1$. There is a surface $S \subset B$ such that $\partial S \subset \partial B$ is homologous to $\beta_{1}-d \ell+k m$ in $\partial B$, where $m$ is a meridian of $\gamma, \ell$ is a longitude of $\gamma$, and $k$ is an integer. Consider simple closed curves $\sigma_{1}$ and $\sigma_{2}$ in $\partial B$ such that $\sigma_{1}=m-d \alpha_{1}$ and $\sigma_{2}=\beta_{1}-d \ell+k m$ in $H_{1}(\partial B)$. Then $\left(\sigma_{1}, \sigma_{2}, \alpha_{2}, \ldots, \alpha_{g}\right)$ is a basis of $\mathcal{L}_{B}^{\mathbb{Z}}$.

Consider the symplectic basis $\left(a_{i}, b_{i}\right)_{1 \leq i \leq g}$ of $H_{1}\left(\partial H_{g} ; \mathbb{Z}\right)$ image of $\left(\alpha_{i}, \beta_{i}\right)_{1 \leq i \leq g}$ by the LP-identification $\partial A \cong \partial H_{g}$. Consider a simple closed curve representing $b_{1}$ in Int $H_{g}$ and a tubular neighborhood $T_{0}$ of this curve. Consider a $d-$ torus $T_{d}$, a symplectic basis $\left(\alpha^{\prime}, \beta^{\prime}\right)$ of $H_{1}\left(\partial T_{d} ; \mathbb{Z}\right)$ and a curve $\gamma^{\prime}$ in $T_{d}$ such that $H_{1}\left(T_{d} ; \mathbb{Z}\right)=(\mathbb{Z} / d \mathbb{Z}) \alpha^{\prime} \oplus \mathbb{Z} \gamma^{\prime}$ and $\beta^{\prime}=d \gamma^{\prime}$. By Lemma 2.6, $\beta^{\prime}$ can be chosen so that $\beta^{\prime}-d \ell\left(\gamma^{\prime}\right)+k m\left(\gamma^{\prime}\right)$ bounds a surface in the exterior of $\gamma^{\prime}$ in $T_{d}$ (where $k$ is the integer that appears when tunneling $A$ ). Choose an LP-identification 
$\partial T_{d} \cong \partial T_{0}$ that identifies $\beta^{\prime}$ with a curve on $\partial T_{0}$ homologous to $b_{1}$ in $H_{g} \backslash \operatorname{Int} T_{0}$. Set $A^{\prime}=H_{g}\left(T_{d} / T_{0}\right)$.

Consider a tunnel $C^{\prime}$ around $\gamma^{\prime}$ in $A^{\prime}$. Set $B^{\prime}=\overline{A^{\prime} \backslash C^{\prime}}$. By Lemma 2.9, $B^{\prime}$ is a $\mathbb{Z} \mathrm{HH}$ of genus $g+1$. Like in $B$, there is a surface $S^{\prime}$ in $B^{\prime}$ bounded by $b_{1}-d \ell\left(\gamma^{\prime}\right)+k m\left(\gamma^{\prime}\right)$, and we can define a basis of $\mathcal{L}_{B^{\prime}}^{\mathbb{Z}}$ similarly. Hence the LPidentification $\partial A \cong \partial H_{g} \cong \partial A^{\prime}$ extends to an LP-identification $\partial B \cong \partial B^{\prime}$. By [1, Lemma 4.11], $B$ can be obtained from $B^{\prime}$ by a finite sequence of Borromean surgeries. Gluing back the cylinders, we get that $A$ can be obtained from $A^{\prime}=H_{g}\left(T_{d} / T_{0}\right)$ by a finite sequence of Borromean surgeries.

Proof of Theorem 1.15 It suffices to prove the result when $B$ is a standard handlebody. We will proceed by induction on $\left|\mathcal{L}_{A}^{T} / \mathcal{L}_{A}^{\mathbb{Z}}\right|$. The case $\left|\mathcal{L}_{A}^{T} / \mathcal{L}_{A}^{\mathbb{Z}}\right|=1$ is given by Corollary 2.12.

Consider a $\mathbb{Q} H H A$ of genus $g$ with $\left|\mathcal{L}_{A}^{T} / \mathcal{L}_{A}^{\mathbb{Z}}\right|>1$, and a standard genus $g$ handlebody $H_{g}$ whose boundary is LP-identified with $\partial A$. By Lemma 2.3, there is a symplectic basis $\left(\alpha_{i}, \beta_{i}\right)_{1 \leq i \leq g}$ of $H_{1}(\partial A ; \mathbb{Z})$, positive integers $d_{i}$, and a basis $\left(\gamma_{i}\right)_{1 \leq i \leq g}$ of $H_{1}(A ; \mathbb{Z}) / \operatorname{Tors}\left(H_{1}(A ; \mathbb{Z})\right)$, such that, in $H_{1}(A ; \mathbb{Z}), d_{i} \alpha_{i}=0$ and $\beta_{i}=d_{i} \gamma_{i}+t_{i}$, with $t_{i} \in \operatorname{Tors}\left(H_{1}(A ; \mathbb{Z})\right)$. Note that $\left|\mathcal{L}_{A}^{T} / \mathcal{L}_{A}^{\mathbb{Z}}\right|=\prod_{1 \leq i \leq g} d_{i}$. Assume $d_{1}>1$.

Consider a tubular neighborhood $T$ of $t_{1}$, with a meridian $m\left(t_{1}\right)$. Consider a $d_{1}-$ torus $T_{d_{1}}$, a basis $(\alpha, \beta)$ of $H_{1}\left(\partial T_{d_{1}} ; \mathbb{Z}\right)$, and a curve $t$ in $T_{d_{1}}$, such that $d_{1} \alpha=0$ and $\beta=d_{1} t$ in $H_{1}\left(T_{d_{1}} ; \mathbb{Z}\right)$. Define an LP-surgery $\left(T_{d_{1}} / T\right)$ by identifying $\alpha$ with $m\left(t_{1}\right)$ and $\beta$ with $t_{1}$. Set $A^{\prime}=A\left(T_{d_{1}} / T\right)$. In $A^{\prime}, t_{1}=d_{1} t$, thus we have $\beta_{1}=d_{1} \gamma$ with $\gamma=\gamma_{1}+t$.

Consider a tunnel $C$ around $\gamma$. Set $B=\overline{A^{\prime} \backslash C}$. By Lemma 2.8, $B$ is a $\mathbb{Q H H}$ of genus $g+1$. There is a surface $S \subset B$ such that $\partial S \subset \partial B$ is homologous to $\beta_{1}-d_{1} \ell+k m$ in $\partial B$, where $m$ is a meridian of $\gamma, \ell$ is a longitude of $\gamma$, and $k$ is an integer. Consider simple closed curves $\sigma_{1}$ and $\sigma_{2}$ in $\partial B$ such that $\sigma_{1}=m-d_{1} \alpha_{1}$ and $\sigma_{2}=\beta_{1}-d_{1} \ell+k m$ in $H_{1}(\partial B)$. The curves $\sigma_{1}$ and $\sigma_{2}$ are null-homologous in $B$, and $\left(\sigma_{1}, \sigma_{2}, \alpha_{2}, \ldots, \alpha_{g}\right)$ is a basis of $\mathcal{L}_{B}^{\mathbb{Z}}$. Hence $\left|\mathcal{L}_{B}^{T} / \mathcal{L}_{B}^{\mathbb{Z}}\right|<\left|\mathcal{L}_{A}^{T} / \mathcal{L}_{A}^{\mathbb{Z}}\right|$.

Consider a genus $g+1$ standard handlebody $H_{g+1}$ of boundary $\partial B$, where the $\sigma_{i}$ and the $\alpha_{i}$ bound disks in $H_{g+1}$. By induction, $B$ can be obtained from $H_{g+1}$ by a finite sequence of elementary surgeries or their inverses. Gluing back the cylinder $C$ to $H_{g+1}$, we get a genus $g \mathbb{Q} H H \tilde{\widetilde{A}}$ satisfying $H_{1}(\widetilde{A})=\left(\mathbb{Z} / d_{1} \mathbb{Z}\right) \alpha_{1} \oplus \mathbb{Z} \gamma \oplus\left(\bigoplus_{2 \leq i \leq g} \mathbb{Z} \beta_{i}\right)$, such that $A^{\prime}$ can be obtained from $\widetilde{A}$ by a finite sequence of elementary surgeries or their inverses. Hence $A$ can be obtained from $\tilde{A}$ by a finite sequence of elementary surgeries or their inverses. Since $\partial \tilde{A}$ and $\partial H_{g}$ are both LP-identified with $\partial A$, they are LP-identified with each other. By Lemma 2.13, $\tilde{A}$ can be obtained from $H_{g}$ by a finite sequence of elementary surgeries or their inverses. 
Remark We could have defined elementary surgeries by restricting the genus 1 case to LP-replacements of standard tori by $p$-tori, for $p$ prime, and keep Theorem 1.15 true. Indeed, consider a $d$-torus $T_{d}$ and the usual curve $\gamma$ in $T_{d}$ that generates $H_{1}\left(T_{d} ; \mathbb{Z}\right) /$ Torsion. One can check that an LP-replacement of a tubular neighborhood of $\gamma$ by a $d^{\prime}$-torus produces a $d d^{\prime}$-torus. Hence, for any positive integer $d$, a $d$-torus $T_{d}$ can be obtained from a standard torus by a finite sequence of "prime" elementary surgeries of genus 1 . Use then the "tunneling method" to see that any $d$-torus can be obtained from this $T_{d}$, with the right choice of longitude, by a finite sequence of Borromean surgeries.

\section{Borromean surgeries and clasper calculus}

Fix a 3-manifold $M$, possibly with boundary. Let $\mathcal{F}_{0}^{\mathbb{Z}}(M)$ denote the rational vector space generated by all the 3-manifolds that can be obtained from $M$ by a finite sequence of Borromean surgeries, up to orientation-preserving homeomorphism. Let $\mathcal{F}_{n}^{\mathbb{Z}}(M)$ denote the subspace generated by the $[M ; \Gamma]$ for all $m$-component Y-link $\Gamma$ in $M$, with $m \geq n$. Let " $={ }_{n}$ " denote the equality modulo $\mathcal{F}_{n+1}^{\mathbb{Z}}(M)$.

Lemma 3.1 [3, Corollary 4.3] Let $\Gamma$ be an $n$-component $Y$-link in a 3-manifold $M$. Let $\ell$ be a leaf of $\Gamma$. Let $\gamma$ be a framed arc starting at the vertex incident to $\ell$ and ending in another point of $\ell$, embedded in $M$ as the core of a band glued to the associated surface of $\Gamma$ as shown in Figure 9. The arc $\gamma$ splits the leaf $\ell$ into two leaves $\ell^{\prime}$ and $\ell^{\prime \prime}$. Denote by $\Gamma^{\prime}$ and $\Gamma^{\prime \prime}$ the $Y$-links obtained from $\Gamma$ by replacing the leaf $\ell$ by $\ell^{\prime}$ and $\ell^{\prime \prime}$ respectively. Then $[M ; \Gamma]={ }_{n}\left[M ; \Gamma^{\prime}\right]+\left[M ; \Gamma^{\prime \prime}\right]$.
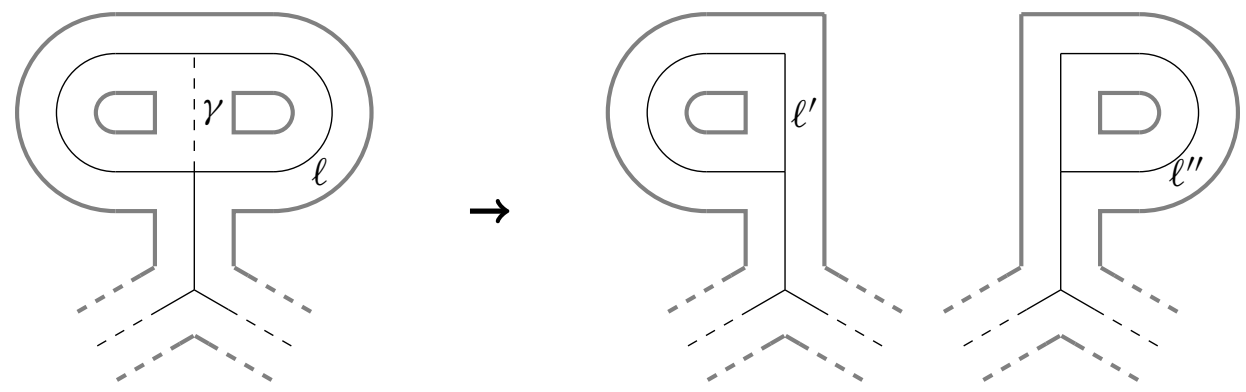

Figure 9: Cutting a leaf

Lemma 3.2 [3, Lemma 4.8] Let $\Gamma$ be an $n$-component $Y$-link in a 3-manifold $M$. If $\Gamma$ has a leaf $\ell$ that bounds a disk in $M \backslash(\Gamma \backslash \ell)$ and has framing 1 , then $[M ; \Gamma]=0$. 
These two lemmas imply that the class of $[M ; \Gamma]$ modulo $\mathcal{F}_{n+1}^{\mathbb{Z}}(M)$ does not depend on the framing of the leaves.

Lemma 3.3 [3, Corollary 4.2] Let $\Gamma$ be an $n$-component $Y$-link in a 3-manifold $M$. Let $K$ be a framed knot in $M \backslash \Gamma$. Let $\Gamma^{\prime}$ be obtained from $\Gamma$ be sliding an edge of $\Gamma$ along $K$ (see Figure 10). Then $[M ; \Gamma]={ }_{n}\left[M ; \Gamma^{\prime}\right]$.

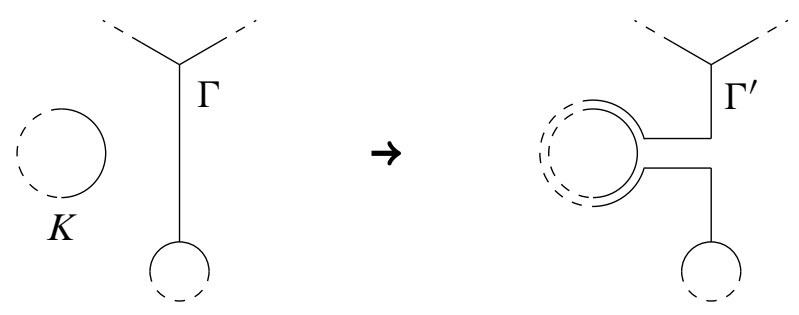

Figure 10: Sliding an edge

Lemma 3.4 [3, Lemma 4.4] Let $\Gamma$ be an $n$-component $Y$-link in a 3-manifold $M$. Let $\Gamma^{\prime}$ be obtained from $\Gamma$ by twisting the framing of an edge by a half twist. Then $\left[M ; \Gamma^{\prime}\right]={ }_{n}-[M ; \Gamma]$.

In the following, we will consider oriented $Y$-links, defined as follows. A Y-graph is oriented if its associated surface is oriented. An orientation of a Y-graph induces an orientation of its leaves and of its internal vertex, as shown in Figure 11, where the surface drawn is given the standard orientation of the plane. A Y-link is oriented if its

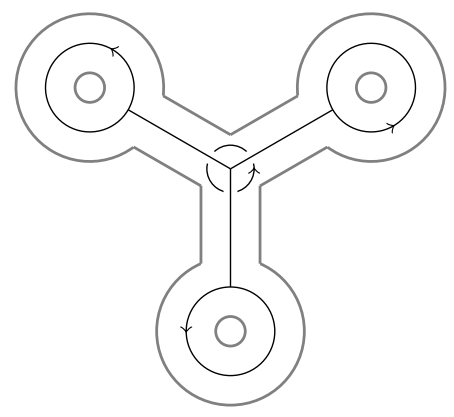

Figure 11: Oriented Y-graph

components are oriented. In this setting, one can twist the framing of an edge only by an integral number of twists. A half twist corresponds to a change of orientation of the adjacent leaf. 
Let $\Gamma$ be an oriented Y-link in a 3-manifold $M$. The above results imply that the class of $[M ; \Gamma]$ modulo $\mathcal{F}_{n+1}^{\mathbb{Z}}(M)$ does not depend on the edges of $\Gamma$ and on the incident vertices of the leaves of $\Gamma$. We shall see that, in some sense, it only depends on the homology classes of the leaves.

Lemma 3.5 [3, Lemma 2.2] Let $\Gamma$ be a $Y$-graph in a 3-manifold $M$, which has a 0 -framed leaf $\ell$ that bounds a disk in $M \backslash(\Gamma \backslash \ell)$. Then $M(\Gamma) \cong M$.

Lemma 3.6 Let $\Gamma$ be an oriented $n$-component $Y$-link in a 3-manifold $M$. Assume $\Gamma$ has a leaf $\ell$ which is trivial in $H_{1}(M \backslash(\Gamma \backslash \ell) ; \mathbb{Z})$. Then $[M ; \Gamma]={ }_{n} 0$.

Proof We can assume that $\ell$ is 0 -framed. The leaf $\ell$ bounds a surface $\Sigma$ whose interior does not meet $\Gamma$. First assume $\Sigma$ has a positive genus. Thanks to Lemma 3.1, we can assume $\Sigma$ has genus 1. Apply Lemma 3.1 to decompose $\ell$ into four leaves, and apply it again to reglue them by pairs, as shown in Figure 12. This leads us to the


Figure 12: Decomposing a leaf

case of a leaf which bounds a disk. The result follows then from Lemma 3.5.

Lemma 3.7 Let $\Gamma$ be an $n$-component $Y$-link in a 3-manifold $M$. Let $\ell$ be a leaf of $\Gamma$. Fix $\Gamma \backslash \ell$. Then the class of $[M ; \Gamma] \bmod \mathcal{F}_{n+1}^{\mathbb{Z}}(M)$ is a linear function of $\ell \in H_{1}(M \backslash(\Gamma \backslash \ell) ; \mathbb{Q})$.

Proof Consider an $n$-component Y-link $\Gamma^{\prime}$ that has a leaf $\ell^{\prime}$ such that $\Gamma^{\prime} \backslash \ell^{\prime}$ coincides with $\Gamma \backslash \ell$ and $\ell^{\prime}$ is homologous to $\ell$ in $M \backslash(\Gamma \backslash \ell)$. Construct another $n$-component $\mathrm{Y}$-link $\Gamma^{\delta}$ by replacing the leaf $\ell$ by $\ell-\ell^{\prime}$ in $\Gamma$ (see Figure 13). By Lemma 3.6, $\left[M ; \Gamma^{\delta}\right]=0$. Thus Lemma 3.1 implies $[M ; \Gamma]={ }_{n}\left[M ; \Gamma^{\prime}\right]$. Hence, for $\Gamma \backslash \ell$ fixed, $[M ; \Gamma] \bmod \mathcal{F}_{n+1}^{\mathbb{Z}}(M)$ only depends on the class of $\ell$ in $H_{1}(M \backslash(\Gamma \backslash \ell) ; \mathbb{Z})$. The linearity follows from Lemma 3.1. Since the $\mathcal{F}_{n}^{\mathbb{Z}}(M)$ are rational vector spaces, $[M ; \Gamma] \bmod \mathcal{F}_{n+1}^{\mathbb{Z}}(M)$ only depends on the rational homology class of $\ell$.

In the case of $\mathbb{Q} H S s$, we want to restrict the set of generators of $\mathcal{F}_{n}^{\mathbb{Z}}(M) / \mathcal{F}_{n+1}^{\mathbb{Z}}(M)$ to brackets defined by Jacobi diagrams.

Lemma 3.3 implies the following. 

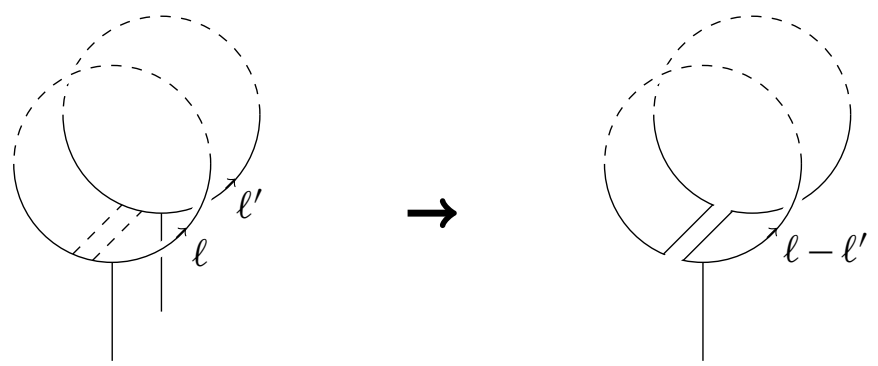

Figure 13: The leaf $\ell-\ell^{\prime}$

Lemma 3.8 Let $J$ be a Jacobi diagram of degree $n / 2$. Equip $J$ with a framing induced by an immersion of $J$ in the plane. Embed the framed diagram $J$ in a 3manifold $M$. Let $\Gamma$ be the oriented $n$-component $Y$-link obtained from $J$ by replacing its edges as shown in Figure 14 . Then the class of $[M ; \Gamma]$ modulo $\mathcal{F}_{n+1}^{\mathbb{Z}}(M)$ does not depend on the embedding and framing of $J$.

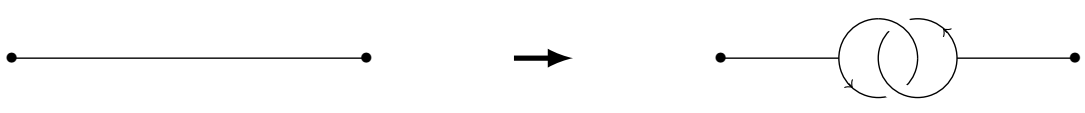

Figure 14: Replacement of an edge

In the sequel, we will denote by $[M ; J]$ the class of $[M ; \Gamma]$ modulo $\mathcal{F}_{n+1}^{\mathbb{Z}}(M)$.

Lemma 3.9 Let $\Gamma$ be an oriented $n$-component $Y$-link in a 3-manifold $M$. Assume that all the leaves of $\Gamma$ are trivial in $H_{1}(M ; \mathbb{Q})$. Then $[M ; \Gamma]$ is equal to a $\mathbb{Q}$-linear combination of terms $[M ; J]$ for some Jacobi diagrams $J$, modulo $\mathcal{F}_{n+1}^{\mathbb{Z}}(M)$.

Proof Suppose $\Gamma$ has a leaf $\ell$ which is nontrivial in $H_{1}(M ; \mathbb{Z})$. Then there is a positive integer $k$ such that $k \ell=0$ in $H_{1}(M ; \mathbb{Z})$. Denote by $\Gamma^{\prime}$ the Y-link obtained from $\Gamma$ by replacing the leaf $\ell$ by a leaf homologous to $k \ell$ in $H_{1}(M \backslash(\Gamma \backslash \ell)$; $\mathbb{Z})$. By Lemma 3.7, we have $[M ; \Gamma]={ }_{n} \frac{1}{k}\left[M ; \Gamma^{\prime}\right]$. Thus we can assume that all the leaves of $\Gamma$ are null-homologous in $M$. As we have seen above, we also can assume that they are 0 -framed.

Such leaves bound embedded surfaces in $M$. Thanks to Lemma 3.3, we can assume that the interior of these surfaces do not meet the edges of $\Gamma$. Consider a leaf $\ell$ of $\Gamma$. Apply Lemma 3.1 to cut $\ell$ into some leaves which are meridians of other leaves, and one leaf which bounds a surface in $M \backslash(\Gamma \backslash \ell)$. The last one can be excluded by applying Lemma 3.6. Cutting similarly each leaf of $\Gamma$, we obtain Y-links whose 
leaves are linked by pairs, in the pattern of Hopf links. Since Lemma 3.7 allows us to change the orientation of a leaf, modulo a sign, we get Y-links obtained from Jacobi diagrams.

Corollary 3.10 Let $M$ be a $\mathbb{Q H S}$. Then $\mathcal{F}_{n}^{\mathbb{Z}}(M) / \mathcal{F}_{n+1}^{\mathbb{Z}}(M)$ is generated by the $[M ; J]$ for all Jacobi diagrams $J$ of degree $n / 2$. In particular, if $n$ is odd, $\mathcal{F}_{n}^{\mathbb{Z}}(M)=$ $\mathcal{F}_{n+1}^{\mathbb{Z}}(M)$.

We end the section by focusing the case of Y-graphs.

Lemma 3.11 Let $\Gamma$ be an oriented $Y$-graph in a 3-manifold $M$. Suppose that $\Gamma$ has two leaves $\ell$ and $\ell^{\prime}$ that bound disks in $M \backslash\left(\Gamma \backslash\left(\ell \cup \ell^{\prime}\right)\right)$ and that form a positive Hopf link. Then $[M ; \Gamma]={ }_{2} 0$.

Proof If the curve $\alpha$ obtained from the leaves $\ell$ and $\ell^{\prime}$ and their adjacent edges, as shown in Figure 15, is 0 -framed and bounds a disk whose interior does not meet $\Gamma$, then, according to [3, Lemma 2.3], the surgery on $\Gamma$ preserves the homeomorphism class of $M$. Lemma 3.3 allows us to reduce the proof to this case.

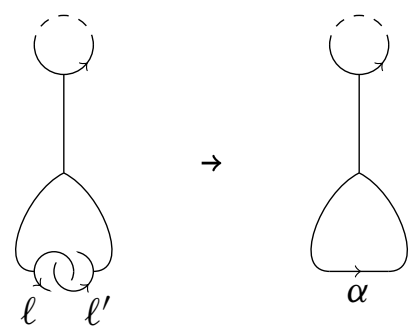

Figure 15: The Y-graph $\Gamma$ and the associated curve $\alpha$

Lemma 3.12 Let $\Gamma$ be an oriented $Y$-graph in a 3-manifold $M$. If $\Gamma$ has a leaf $\ell$ which is trivial in $H_{1}(M ; \mathbb{Q})$, then $[M ; \Gamma]={ }_{2} 0$.

Proof As in the proof of Lemma 3.9, we can assume that $\ell$ is null-homologous in $M$ and 0 -framed. Then $\ell$ bounds a surface $\Sigma$. Using Lemma 3.3, we can assume that its interior $\stackrel{\circ}{\Sigma}$ does not meet the edges of $\Gamma$. However, it can meet the other leaves. Using Lemma 3.1 to decompose the different leaves of $\Gamma$, we can restrict to two cases. Either $\stackrel{\circ}{\Sigma}$ does not meet $\Gamma$, or $\ell$ is linked with another leaf in the pattern of a Hopf link. Conclude with Lemma 3.6 in the first case. In the second case, since Lemma 3.7 allows us to change the orientation of a leaf, modulo a sign, conclude with Lemma 3.11. 
Lemma 3.13 Let $\Gamma$ be an oriented $Y$-graph in a 3-manifold $M$. The class of $[M ; \Gamma]$ modulo $\mathcal{F}_{2}^{\mathbb{Z}}(M)$ only depends on the classes of the leaves of $\Gamma$ in $H_{1}(M ; \mathbb{Q})$. Moreover, the dependance is trilinear and alternating.

Proof Consider a leaf $\ell$ of $\Gamma$. Consider an oriented Y-graph $\Gamma^{\prime}$ and a leaf $\ell^{\prime}$ of $\Gamma^{\prime}$ such that $\Gamma^{\prime} \backslash \ell^{\prime}$ coincides with $\Gamma \backslash \ell$ and $\ell^{\prime}=\ell$ in $H_{1}(M ; \mathbb{Q})$. Construct another Y-graph $\Gamma^{\delta}$ by replacing the leaf $\ell$ by $\ell-\ell^{\prime}$ in $\Gamma$ (see Figure 13). By Lemma 3.12, $\left[M ; \Gamma^{\delta}\right]=0$. Thus Lemma 3.1 implies $[M ; \Gamma]={ }_{n}\left[M ; \Gamma^{\prime}\right]$. Hence, for $\Gamma \backslash \ell$ fixed, $[M ; \Gamma] \bmod \mathcal{F}_{2}^{\mathbb{Z}}(M)$ only depends on the class of $\ell$ in $H_{1}(M ; \mathbb{Q})$. The linearity follows from Lemma 3.1. To get the alternating property, note that exchanging two leaves is equivalent to changing the orientation of the three leaves.

\section{Finite type invariants of degree 1}

\subsection{The family $\left(M_{p}-S^{3}\right)_{p}$ prime generates $\mathcal{G}_{1}$}

We denote by " $={ }_{2}$ " the equality modulo $\mathcal{F}_{2}$. Note that $\mathcal{F}_{1}$ is generated by the $\left(M-S^{3}\right)$. For any $\mathbb{Q H S} M$, let $l k_{M}: H_{1}(M ; \mathbb{Z}) \times H_{1}(M ; \mathbb{Z}) \rightarrow \mathbb{Q} / \mathbb{Z}$ be the linking form on $H_{1}(M ; \mathbb{Z})$.

Lemma 4.1 Let $M$ and $N$ be $\mathbb{Q H S s}$ such that $\left(H_{1}(M ; \mathbb{Z}), l k_{M}\right) \cong\left(H_{1}(N ; \mathbb{Z}), l k_{N}\right)$. Then $M={ }_{2} N$.

Proof By [12, Theorem 2], $N$ can be obtained from $M$ by a finite sequence of Borromean surgeries. It suffices to show that $M\left(B^{\prime} / B\right)={ }_{2} M$ for one Borromean surgery $\left(B^{\prime} / B\right)$. This follows from Corollary 3.10.

We call linking a pair $(H, \phi)$, where $H$ is a finite abelian group, and $\phi$ is a nondegenerate symmetric bilinear form on $H$, with values in $\mathbb{Q} / \mathbb{Z}$. Consider the abelian semigroup $\mathfrak{N}$ of all linkings under orthogonal sum. We have a homomorphism $\mathcal{H}$ from the semigroup of all $\mathbb{Q} H S s$ under connected sum to $\mathfrak{N}$, given by $\mathcal{H}(M)=\left(H_{1}(M ; \mathbb{Z}), l k_{M}\right)$. By Kawauchi and Kojima [5, Theorem 6.1], this homomorphism is onto. So we can define an equivalence relation on $\mathfrak{N}$ by $H_{1} \sim_{2} H_{2}$ if $H_{1}=\mathcal{H}\left(M_{1}\right), H_{2}=\mathcal{H}\left(M_{2}\right)$, and $M_{1}-S^{3}={ }_{2} M_{2}-S^{3}$.

Note that

$$
M \sharp N-S^{3}={ }_{2}\left(M-S^{3}\right)+\left(N-S^{3}\right) .
$$

Thus, by Lemma 4.1, in order to prove that $\left(M_{p}-S^{3}\right)_{p}$ prime generates $\mathcal{G}_{1}$, it suffices to show that any $H \in \mathfrak{N}$ is 2 -equivalent to a direct sum of groups $\mathbb{Z}_{p}:=\mathbb{Z} / p \mathbb{Z}$, with 
$p$ prime, independently of the associated bilinear form. Since $\mathfrak{N}$ is the direct sum of the abelian semigroups $\mathfrak{N}_{p}$ of linkings on $p$-groups, we restrict ourselves to the study of $p$-groups.

Lemma 4.2 Any linking in $\mathfrak{N}_{p}$ is 2-equivalent to an orthogonal sum of linkings on cyclic $p$-groups. Two linkings defined on the same cyclic group are 2-equivalent.

Proof In the case of odd primes $p$, by Wall [16, Theorem 4], $\mathfrak{N}_{p}$ has generators $A_{p^{k}}$, $B_{p^{k}}, k \geq 1$, and sole relation $2 A_{p^{k}}=2 B_{p^{k}}\left(\mathcal{R}_{p^{k}}\right)$, where

- $A_{p^{k}}=\left(\mathbb{Z}_{p^{k}}, \phi_{A}\right), \phi_{A}(1,1)=1 / p^{k}$

- $\quad B_{p^{k}}=\left(\mathbb{Z}_{p^{k}}, \phi_{B}\right), \phi_{B}(1,1)=x / p^{k}$ with $x$ nonsquare modulo $p^{k}$.

The relations $(\star)$ and $\left(\mathcal{R}_{p^{k}}\right)$ show that $A_{p^{k}} \sim_{2} B_{p^{k}}$.

In the case of 2-groups, we will use the presentation of $\mathfrak{N}_{2}$ given by Miranda in the introduction of [15], which is an alternative version of the description of $\mathfrak{N}_{2}$ obtained in [5]. The generators are 4 linkings $A_{k}, B_{k}, C_{k}, D_{k}$, defined on $\mathbb{Z}_{2^{k}}$, and 2 linkings $E_{k}, F_{k}$, defined on $\mathbb{Z}_{2^{k}} \times \mathbb{Z}_{2^{k}}$. The relation $A_{k}+E_{k}=2 A_{k}+B_{k}$ implies $E_{k} \sim_{2} A_{k}+B_{k}$, and the relation $2 E_{k}=2 F_{k}$ implies $E_{k} \sim_{2} F_{k}$. So we are lead to the cyclic case. The relations $2 A_{k}=2 C_{k}, 2 B_{k}=2 D_{k}, 4 A_{k}=4 B_{k}$, give $A_{k} \sim_{2} B_{k} \sim_{2} C_{k} \sim_{2} D_{k}$.

Lemma 4.2 reduces our study to the case of cyclic groups with arbitrary linkings.

Lemma 4.3 Denote by $G_{p^{k}}$ any linking on $\mathbb{Z}_{p^{k}}$. We have $G_{p^{k+k^{\prime}}} \sim_{2} G_{p^{k}}+G_{p^{k^{\prime}}}$ for any prime $p$ and any positive integers $k$ and $k^{\prime}$. It follows that $G_{p^{k}} \sim_{2} k G_{p}$.

Proof We will use the following easy result.

Sublemma 4.4 Let $d$ be a positive integer. Let $T_{d}$ be a $d$-torus. Let $(\alpha, \beta)$ be a symplectic basis of $H_{1}\left(\partial T_{d} ; \mathbb{Z}\right)$ such that $\alpha$ generates $\mathcal{L}_{T_{d}}$ and $\beta=d \gamma$ in $H_{1}\left(T_{d} ; \mathbb{Z}\right)$. Let $T$ be a standard solid torus trivially embedded in $S^{3}$. Define an LP-identification $\partial T_{d} \cong \partial T$ that identifies $\beta$ with the preferred longitude of $T$. Then $H_{1}\left(S^{3}\left(T_{d} / T\right)\right)=$ $\mathbb{Z}_{d} \alpha \oplus \mathbb{Z}_{d} \gamma$.

In $S^{3}$, consider two disjoint, trivially embedded, tori $T$ and $T^{\prime}$, linked in the pattern of a Hopf link. Consider the LP-surgeries given by Sublemma 4.4 for $d=p^{k}$ and for $d=p^{k^{\prime}}$. We still denote by $\alpha, \beta, \gamma$ (respectively $\alpha^{\prime}, \beta^{\prime}, \gamma^{\prime}$ ) the curves defined in the lemma. We have

$$
H_{1}\left(S^{3}\left(\frac{T_{p^{k}}}{T}\right)\right)=\mathbb{Z}_{p^{k}} \alpha \times \mathbb{Z}_{p^{k}} \gamma, \quad H_{1}\left(S^{3}\left(\frac{T_{p^{k^{\prime}}}}{T^{\prime}}\right)\right)=\mathbb{Z}_{p^{k^{\prime}}} \alpha^{\prime} \times \mathbb{Z}_{p^{k^{\prime}}} \gamma^{\prime} .
$$


Now, in $S^{3}\left(T_{p^{k}} / T, T_{p^{k^{\prime}}} / T^{\prime}\right)$, we have $\alpha^{\prime}=\beta=p^{k} \gamma$ and $\alpha=\beta^{\prime}=p^{k^{\prime}} \gamma^{\prime}$. Thus

$$
H_{1}\left(S^{3}\left(\frac{T_{p^{k}}}{T}, \frac{T_{p^{k^{\prime}}}}{T^{\prime}}\right)\right)=\mathbb{Z}_{p^{k+k^{\prime}}} \gamma \times \mathbb{Z}_{p^{k+k^{\prime}}} \gamma^{\prime}
$$

Conclude with the following equality:

$$
S^{3}\left(\frac{T_{p^{k}}}{T}, \frac{T_{p^{k^{\prime}}}}{T^{\prime}}\right)-S^{3}={ }_{2}\left(S^{3}\left(\frac{T_{p^{k}}}{T}\right)-S^{3}\right)+\left(S^{3}\left(\frac{T_{p^{k^{\prime}}}}{T^{\prime}}\right)-S^{3}\right) .
$$

This achieves the proof of the first part of Proposition 1.8, namely the fact that the family $\left(M_{p}-S^{3}\right)_{p}$ prime generates $\mathcal{G}_{1}$.

\subsection{The invariants $v_{p}$}

In this subsection, unless otherwise mentioned, all the homology modules are considered with integral coefficients. We prove the following proposition that implies Proposition 1.9.

Proposition 4.5 Consider a $\mathbb{Q H S} M$, two disjoint genus $g \mathbb{Q H H s} A$ and $B$ in $M$, and two $\mathbb{Q H H s} A^{\prime}$ and $B^{\prime}$ whose boundaries are LP-identified with $\partial A$ and $\partial B$ respectively. Then

$$
\frac{\left|H_{1}(M)\right|}{\left|H_{1}\left(M\left(A^{\prime} / A\right)\right)\right|}=\frac{\left|H_{1}\left(M\left(B^{\prime} / B\right)\right)\right|}{\left|H_{1}\left(M\left(A^{\prime} / A, B^{\prime} / B\right)\right)\right|} .
$$

Proof The exact sequence associated with $(M, A)$ gives

$$
0 \rightarrow H_{2}(M, A) \rightarrow H_{1}(A) \rightarrow H_{1}(M) \rightarrow H_{1}(M, A) \rightarrow 0 .
$$

Set $X=M \backslash \operatorname{Int} A$. By excision, we have $H_{i}(M, A)=H_{i}(X, \partial X)$ for any integer $i$. So the above exact sequence can be rewritten as follows:

$$
0 \rightarrow H_{2}(X, \partial X) \stackrel{\varphi_{1}}{\longrightarrow} H_{1}(A) \stackrel{\varphi_{2}}{\longrightarrow} H_{1}(M) \stackrel{\varphi_{3}}{\longrightarrow} H_{1}(X, \partial X) \rightarrow 0 .
$$

Since $H_{1}(M)$ is finite, $H_{1}(X, \partial X)$ also is, and we have $\left|H_{1}(M)\right|=\left|H_{1}(X, \partial X)\right|$. $\left|\operatorname{Im} \varphi_{2}\right|$.

Similarly, we have an exact sequence

$$
0 \rightarrow H_{2}(X, \partial X) \stackrel{\varphi_{1}^{\prime}}{\longrightarrow} H_{1}\left(A^{\prime}\right) \stackrel{\varphi_{2}^{\prime}}{\longrightarrow} H_{1}\left(M\left(A^{\prime} / A\right)\right) \stackrel{\varphi_{3}^{\prime}}{\longrightarrow} H_{1}(X, \partial X) \rightarrow 0 .
$$

We get

$$
\frac{\left|H_{1}(M)\right|}{\left|H_{1}\left(M\left(A^{\prime} / A\right)\right)\right|}=\frac{\left|\operatorname{Im} \varphi_{2}\right|}{\left|\operatorname{Im} \varphi_{2}^{\prime}\right|} .
$$


Similarly arguing with $M\left(B^{\prime} / B\right)$ instead of $M$, and setting $X^{\prime}=X\left(B^{\prime} / B\right)$, we have the exact sequences

$$
\begin{gathered}
0 \rightarrow H_{2}\left(X^{\prime}, \partial X^{\prime}\right) \stackrel{\psi_{1}}{\longrightarrow} H_{1}(A) \stackrel{\psi_{2}}{\longrightarrow} H_{1}\left(M\left(B^{\prime} / B\right)\right) \stackrel{\psi_{3}}{\longrightarrow} H_{1}\left(X^{\prime}, \partial X^{\prime}\right) \rightarrow 0, \\
0 \rightarrow H_{2}\left(X^{\prime}, \partial X^{\prime}\right) \stackrel{\psi_{1}^{\prime}}{\longrightarrow} H_{1}\left(A^{\prime}\right) \stackrel{\psi_{2}^{\prime}}{\longrightarrow} H_{1}\left(M\left(A^{\prime} / A, B^{\prime} / B\right)\right) \stackrel{\psi_{3}^{\prime}}{\longrightarrow} H_{1}\left(X^{\prime}, \partial X^{\prime}\right) \rightarrow 0,
\end{gathered}
$$

and we get

$$
\frac{\left|H_{1}\left(M\left(B^{\prime} / B\right)\right)\right|}{\left|H_{1}\left(M\left(A^{\prime} / A, B^{\prime} / B\right)\right)\right|}=\frac{\left|\operatorname{Im} \psi_{2}\right|}{\left|\operatorname{Im} \psi_{2}^{\prime}\right|} .
$$

We now relate $\left|\operatorname{Im} \varphi_{2}\right|$ and $\left|\operatorname{Im} \psi_{2}\right|$. Since $\operatorname{Im} \varphi_{2} \cong H_{1}(A) / \operatorname{Im} \varphi_{1}$ and $\operatorname{Im} \psi_{2} \cong$ $H_{1}(A) / \operatorname{Im} \psi_{1}$, we shall study $\operatorname{Im} \varphi_{1}$ and $\operatorname{Im} \psi_{1}$.

The following sublemma gives us additional information about $X$.

Sublemma 4.6 If $M$ is a $\mathbb{Q H S}$ and if $A$ is a genus $g \mathbb{Q H H}$ in $M$, then $X=M \backslash \operatorname{Int} A$ also is a genus $g \mathbb{Q H H}$.

Proof It is clear that $H_{3}(X ; \mathbb{Q})=0$ and $H_{0}(X ; \mathbb{Q})=\mathbb{Q}$.

The Mayer-Vietoris sequence associated with $M=A \cup X$ gives

$$
0 \rightarrow H_{3}(M ; \mathbb{Q}) \rightarrow H_{2}(\partial A ; \mathbb{Q}) \rightarrow H_{2}(A ; \mathbb{Q}) \oplus H_{2}(X ; \mathbb{Q}) \rightarrow 0 .
$$

Since $H_{3}(M ; \mathbb{Q}) \rightarrow H_{2}(\partial A ; \mathbb{Q})$ is an isomorphism that identifies the fundamental classes, we have $H_{2}(X ; \mathbb{Q})=0$.

The Mayer-Vietoris sequence also gives an isomorphism $H_{1}(\partial A ; \mathbb{Q}) \cong H_{1}(A ; \mathbb{Q}) \oplus$ $H_{1}(X ; \mathbb{Q})$, thus $H_{1}(X ; \mathbb{Q})=\mathbb{Q}^{g}$.

We have the following commutative diagram, where $i_{\star}$ is the map induced by the inclusion $i: \partial A \hookrightarrow A$. Denote the images of $H_{2}(X, \partial X)$ and $H_{2}\left(X^{\prime}, \partial X^{\prime}\right)$ in $H_{1}(\partial A)$

$$
\begin{aligned}
& H_{2}(X, \partial X) \stackrel{\partial}{\longrightarrow} H_{1}(\partial A) \stackrel{\partial}{\longleftarrow} H_{2}\left(X^{\prime}, \partial X^{\prime}\right) \\
& \varphi_{1} \quad \underset{H_{1}(A)}{\downarrow} i_{\star} / \psi_{1}
\end{aligned}
$$

by $F$ and $F^{\prime}$ respectively. Since $\varphi_{1}$ and $\psi_{1}$ are injective, the two boundary operators also are. Thus, by Sublemma 4.6 and Lemma 2.2, $F$ and $F^{\prime}$ are free submodules of $H_{1}(\partial A)$, of rank $g$. Consider bases $\gamma$ of $F$ and $\gamma^{\prime}$ of $F^{\prime}$. Over $\mathbb{Q}, F$ generates the Lagrangian $\mathcal{L}_{X}$, and $F^{\prime}$ generates $\mathcal{L}_{X^{\prime}}$. Since $X^{\prime}$ is obtained from $X$ by an 
LP-surgery, we have $\mathcal{L}_{X}=\mathcal{L}_{X^{\prime}}$. Hence we have a matrix $R \in G L_{g}(\mathbb{Q})$ of change of basis from $\gamma$ to $\gamma^{\prime}$. Thus

$$
\left|\operatorname{Im} \psi_{2}\right|=\left|\frac{H_{1}(A)}{\operatorname{Im} \psi_{1}}\right|=\left|\frac{H_{1}(A)}{i_{\star}\left(F^{\prime}\right)}\right|=|\operatorname{det} R| \cdot\left|\frac{H_{1}(A)}{i_{\star}(F)}\right|=|\operatorname{det} R| \cdot\left|\operatorname{Im} \varphi_{2}\right| .
$$

Since the same submodules $F$ and $F^{\prime}$ occur in the decomposition of $\varphi_{1}^{\prime}$ and $\psi_{1}^{\prime}$, we also have

$$
\left|\operatorname{Im} \psi_{2}^{\prime}\right|=|\operatorname{det} R| \cdot\left|\operatorname{Im} \varphi_{2}^{\prime}\right|
$$

Finally,

$$
\frac{\left|H_{1}\left(M\left(B^{\prime} / B\right)\right)\right|}{\left|H_{1}\left(M\left(A^{\prime} / A, B^{\prime} / B\right)\right)\right|}=\frac{\left|\operatorname{Im} \psi_{2}\right|}{\left|\operatorname{Im} \psi_{2}^{\prime}\right|}=\frac{|\operatorname{det} R| \cdot\left|\operatorname{Im} \varphi_{2}\right|}{|\operatorname{det} R| \cdot\left|\operatorname{Im} \varphi_{2}^{\prime}\right|}=\frac{\left|H_{1}(M)\right|}{\left|H_{1}\left(M\left(A^{\prime} / A\right)\right)\right|} .
$$

\section{Additive invariants of degree $n>1$}

\subsection{Degree 1 invariants of framed rational homology tori}

Fix a genus 1 surface $\Sigma_{1}$ and a symplectic basis $\left(\alpha_{0}, \beta_{0}\right)$ of $H_{1}\left(\Sigma_{1} ; \mathbb{Z}\right)$. Define $\mathcal{F}_{0}\left(\Sigma_{1}\right)$ as the rational vector space generated by all the rational homology tori $T$, equipped with an oriented longitude $\ell(T)$. Denote by $m(T)$ the meridian of $T$ that satisfies $\langle m(T), \ell(T)\rangle_{\partial T}=1$. The data of the framing is equivalent to the data of an orientation-preserving homeomorphism $h: \Sigma_{1} \rightarrow \partial T$ such that $h_{*}\left(\mathbb{Q} \alpha_{0}\right)=\mathcal{L}_{T}$, the equivalence being given by $m(T)=h\left(\alpha_{0}\right)$ and $\ell(T)=h\left(\beta_{0}\right)$. In particular, given two framed rational homology tori, we have a canonical LP-identification of their boundaries, which identifies the fixed longitudes. Define a filtration $\left(\mathcal{F}_{n}\left(\Sigma_{1}\right)\right)_{n \in \mathbb{N}}$, and quotients $\left(\mathcal{G}_{n}\left(\Sigma_{1}\right)\right)_{n \in \mathbb{N}}$, as in the case of $\mathbb{Q}$ HSs. Note that $\mathcal{G}_{0}\left(\Sigma_{1}\right) \cong \mathbb{Q}$.

Denote by $T_{0}$ the standard solid torus with a fixed longitude $\ell\left(T_{0}\right)$. For any prime $p$, fix a $\mathbb{Q} H S M_{p}$ such that $H_{1}\left(M_{p} ; \mathbb{Z}\right) \cong \mathbb{Z} / p \mathbb{Z}$. Define a rational homology ball $B_{p}$ by removing an open ball from $M_{p}$. In this subsection, we prove the following.

Proposition 5.1 $\mathcal{G}_{1}\left(\Sigma_{1}\right)=\bigoplus_{p \text { prime }} \mathbb{Q}\left[T_{0} ; \frac{B_{p}}{B^{3}}\right]$

Consider a framed rational homology torus $T$. Set $d(T)=\left|\mathcal{L}_{T}^{T} / \mathcal{L}_{T}^{\mathbb{Z}}\right|$ (see Lemma 2.3 for the definition of $\mathcal{L}_{T}^{T}$ and $\mathcal{L}_{T}^{\mathbb{Z}}$ ). For $p$ prime, define

$$
\mu_{p}(T)=v_{p}\left(d(T)\left|\operatorname{Tors}\left(H_{1}(T ; \mathbb{Z})\right)\right|\right),
$$

where $v_{p}$ denotes the $p$-adic valuation. 
Lemma 5.2 For any prime $p, \mu_{p}$ is a degree 1 invariant of the framed rational homology tori.

Proof Consider a framed rational homology torus $T$. Define a $\mathbb{Q H S} M(T)$ by gluing $T$ and the standard torus $T_{0}$ along their boundaries, in such a way that $\ell(T)$ is identified with $m\left(T_{0}\right)$. We have $H_{1}(M(T))=H_{1}(T) / \mathbb{Z} \ell(T)$. By Lemma 2.3, $\left|H_{1}(M(T))\right|=d(T)\left|\operatorname{Tors}\left(H_{1}(T)\right)\right|$. Thus $\mu_{p}(T)=v_{p}\left(\left|H_{1}(M(T))\right|\right)=v_{p}(M(T))$. The result follows from the fact that $v_{p}$ is a degree 1 invariant of $\mathbb{Q H S s}$.

Corollary 5.3 The sum $\bigoplus_{p \text { prime }} \mathbb{Q}\left[T_{0} ; B_{p} / B^{3}\right]$ is direct.

Lemma 5.4 The space $\mathcal{G}_{1}\left(\Sigma_{1}\right)$ is generated by the $\left[T ; E^{\prime} / E\right]$, where $\left(E^{\prime} / E\right)$ is an elementary surgery.

Proof Consider $\left[T ; A^{\prime} / A\right] \in \mathcal{F}_{1}\left(\Sigma_{1}\right)$. By Theorem 1.15, $A^{\prime}$ is obtained from $A$ by a sequence of elementary surgeries, or their inverses, $\left(E_{i}^{\prime} / E_{i}\right)_{1 \leq i \leq k}$. Set $A_{i}=$ $A\left(E_{1}^{\prime} / E_{1}\right)\left(E_{2}^{\prime} / E_{2}\right) \cdots\left(E_{i}^{\prime} / E_{i}\right)$. Then

$$
\left[T ; \frac{A^{\prime}}{A}\right]=\sum_{i=0}^{k-1}\left[T\left(\frac{A_{i}}{A}\right) ; \frac{A_{i+1}}{A_{i}}\right]=\sum_{i=0}^{k-1}\left[T\left(\frac{A_{i}}{A}\right) ; \frac{E_{i+1}^{\prime}}{E_{i+1}}\right] .
$$

Now, for any $\left[T ; E^{\prime} / E\right] \in \mathcal{F}_{1}\left(\Sigma_{1}\right)$, we have $\left[T ; E^{\prime} / E\right]=-\left[T\left(E^{\prime} / E\right) ; E / E^{\prime}\right]$.

We shall get rid of the elementary surgeries of genus 1 with the help of the following two lemmas.

Lemma 5.5 Let $E$ be a framed standard torus. Let $E^{\prime}$ be a framed $d$-torus. Assume $\ell\left(E^{\prime}\right)=d \gamma$ in $H_{1}\left(E^{\prime} ; \mathbb{Z}\right)$ for a curve $\gamma$ in $E^{\prime}$. Embed two disjoint copies $E_{1}$ and $E_{2}$ of $E$ in Int $E$ so that $\ell\left(E_{1}\right)=\ell\left(E_{2}\right)=\ell(E)$ in $H_{1}\left(E \backslash \operatorname{Int}\left(E_{1} \cup E_{2}\right)\right.$; $\left.\mathbb{Z}\right)$. Let $E_{1}^{\prime}$ and $E_{2}^{\prime}$ be two copies of $E^{\prime}$. Set $A=E\left(E_{1}^{\prime} / E_{1}, E_{2}^{\prime} / E_{2}\right)$. Then there is a $\mathbb{Q H S} M$ such that $A$ can be obtained from $E^{\prime} \sharp M$ by a finite sequence of Borromean surgeries.

Proof For $i=1,2$, denote by $\gamma_{i}$ the copy of $\gamma$ in $E_{i}^{\prime}$, so that $\ell\left(E_{i}^{\prime}\right)=d \gamma_{i}$ in $H_{1}\left(E_{i}^{\prime} ; \mathbb{Z}\right)$. Note that, in $H_{1}(A), \ell(A)=\ell\left(E_{1}^{\prime}\right)=\ell\left(E_{2}^{\prime}\right)$, and $m(A)=m\left(E_{1}^{\prime}\right)+$ $m\left(E_{2}^{\prime}\right)$. We have

$$
\begin{aligned}
H_{1}(A ; \mathbb{Z}) & =\left\langle m\left(E_{1}^{\prime}\right), m\left(E_{2}^{\prime}\right), \gamma_{1}, \gamma_{2} \mid d m\left(E_{1}^{\prime}\right)=0, d m\left(E_{2}^{\prime}\right)=0, d \gamma_{1}=d \gamma_{2}\right\rangle \\
& =\left\langle m\left(E_{1}^{\prime}\right), m(A), \gamma_{1}, \gamma_{2}-\gamma_{1} \mid d m\left(E_{1}^{\prime}\right)=0, d m(A)=0, d\left(\gamma_{2}-\gamma_{1}\right)=0\right\rangle \\
& =\mathbb{Z}_{d} m(A) \oplus \mathbb{Z} \gamma_{1} \oplus \mathbb{Z}_{d} m\left(E_{1}^{\prime}\right) \oplus \mathbb{Z}_{d}\left(\gamma_{2}-\gamma_{1}\right) .
\end{aligned}
$$


Note that $\ell(A)=d \gamma_{1}$. Consider a tunnel $C$ around $\gamma_{1}$. Set $B=\overline{A \backslash C}$. There is a surface $S \subset B$ such that $\partial S \subset \partial B$ is homologous to $\ell(A)-d \ell+k m$ in $\partial B$, where $m$ is a meridian of $\gamma_{1}, \ell$ is a longitude of $\gamma_{1}$, and $k$ is an integer. Consider simple closed curves $\sigma_{1}, \sigma_{2}, \mu_{1}$ and $\mu_{2}$ in $\partial B$ such that $\sigma_{1}=m-d m(A), \sigma_{2}=\ell(A)-d \ell+k m$, $\mu_{1}=-\ell+k m(A)$ and $\mu_{2}=-m(A)$ in $H_{1}(\partial B)$. The curves $\sigma_{1}$ and $\sigma_{2}$ bound embedded surfaces in $B$, and $\left(\sigma_{1}, \mu_{1}, \sigma_{2}, \mu_{2}\right)$ is a symplectic basis of $H_{1}(\partial B ; \mathbb{Z})$. Thus $B$ is a genus $2 \mathbb{Q H H}$ with $\mathcal{L}_{B}^{T} / \mathcal{L}_{B}^{\mathbb{Z}}=0$. By Corollary 2.12 , there are a standard genus 2 handlebody $H_{2}$ and a $\mathbb{Q H S} M$ such that $B$ is obtained from $H_{2} \sharp M$ by a finite sequence of Borromean surgeries.

Now consider the $d$-torus $E^{\prime}$. It is homeomorphic to $E\left(E_{1}^{\prime} / E_{1}\right)$. Consider a tunnel $C^{\prime}$ around $\gamma$ in $E^{\prime}$. We can choose a meridian $m^{\prime}$ and a longitude $\ell^{\prime}$ of $\gamma$ in such a way that there are curves $\sigma_{1}^{\prime}$ and $\sigma_{2}^{\prime}$ on the boundary of $B^{\prime}=\overline{E^{\prime} \backslash C^{\prime}}$ which bound surfaces in $B^{\prime}$ and which are respectively homologous to $m^{\prime}-d \alpha$ and $\beta-d \ell^{\prime}+k m^{\prime}$ in $H_{1}\left(\partial B^{\prime} ; \mathbb{Z}\right)$. Thus the LP-identification $\partial E^{\prime} \cong \partial A$ extends to an LP-identification $\partial B^{\prime} \cong \partial B \cong \partial H_{2}$. Since $H_{1}\left(B^{\prime} ; \mathbb{Z}\right)=\mathbb{Z}\left(\ell^{\prime}-k \alpha\right) \oplus \mathbb{Z} m^{\prime}, B^{\prime}$ and $H_{2}$ are two $\mathbb{Z} H$ whose boundaries are LP-identified. By [1, Lemma 4.11], $H_{2}$ can be obtained from $B^{\prime}$ by a finite sequence of Borromean surgeries. Thus $H_{2} \sharp M$, and $B$, can be obtained from $B^{\prime} \sharp M$ by a finite sequence of Borromean surgeries. Gluing back the cylinders, we see that $A$ can be obtained from $E^{\prime} \sharp M$ by a finite sequence of Borromean surgeries.

Lemma 5.6 The quotient $\mathcal{G}_{1}\left(\Sigma_{1}\right)$ is generated by the $\left[T ; E^{\prime} / E\right]$, where $\left(E^{\prime} / E\right)$ is an elementary surgery of genus 0 (connected sum) or 3 (Borromean surgery).

Proof Consider a framed rational homology torus $T$ and an elementary surgery $E^{\prime} / E$ of genus 1 in $T$, ie, $E$ is an embedded standard torus, and $E^{\prime}$ is a $d$-torus. Fix a longitude $\ell\left(E^{\prime}\right)$ such that $\ell\left(E^{\prime}\right)=d \gamma$ in $H_{1}\left(E^{\prime} ; \mathbb{Z}\right)$ for a curve $\gamma$ in $E^{\prime}$. Choose the longitude $\ell(E)$ which is identified with $\ell\left(E^{\prime}\right)$ by the LP-identification $\partial E \cong \partial E^{\prime}$.

Consider the copies $E_{1}$ and $E_{2}$ of $E$ in Int $E$, the copies $E_{1}^{\prime}$ and $E_{2}^{\prime}$ of $E^{\prime}$, the rational homology torus $A$, and the $\mathbb{Q H S} M$, defined in Lemma 5.5. Set $T^{\prime}=$ $T\left(E_{1}^{\prime} / E_{1}, E_{2}^{\prime} / E_{2}\right) \cong T(A / E)$. Write

$$
A=E^{\prime}\left(\frac{B(M)}{B^{3}}\right)\left(\frac{B_{1}^{\prime}}{B_{1}}\right)\left(\frac{B_{2}^{\prime}}{B_{2}}\right) \cdots\left(\frac{B_{k}^{\prime}}{B_{k}}\right),
$$

where $B(M)$ is the rational homology ball obtained by removing a ball $B^{3}$ from $M$, and the $\left(B_{i}^{\prime} / B_{i}\right)$ are Borromean surgeries. On the one hand, we have

$$
\left[T ; \frac{E_{1}^{\prime}}{E_{1}}, \frac{E_{2}^{\prime}}{E_{2}}\right]=2\left[T ; \frac{E^{\prime}}{E}\right]-T+T^{\prime},
$$


thus

$$
T-T^{\prime}=2\left[T ; \frac{E^{\prime}}{E}\right] \bmod \mathcal{F}_{2}\left(\Sigma_{1}\right)
$$

On the other hand,

$$
\begin{aligned}
T-T^{\prime}=\left[T ; \frac{A}{E}\right]=\left[T ; \frac{E^{\prime}}{E}\right] & +\left[T\left(\frac{E^{\prime}}{E}\right) ; \frac{B(M)}{B^{3}}\right] \\
& +\sum_{i=1}^{k}\left[T\left(\frac{E^{\prime}}{E}\right)\left(\frac{B(M)}{B^{3}}\right)\left(\frac{B_{1}^{\prime}}{B_{1}}\right) \cdots\left(\frac{B_{i-1}^{\prime}}{B_{i-1}}\right) ; \frac{B_{i}^{\prime}}{B_{i}}\right] .
\end{aligned}
$$

Thus,

$$
\begin{aligned}
{\left[T ; \frac{E^{\prime}}{E}\right]=} & {\left[T\left(\frac{E^{\prime}}{E}\right) ; \frac{B(M)}{B^{3}}\right] } \\
& +\sum_{i=1}^{k}\left[T\left(\frac{E^{\prime}}{E}\right)\left(\frac{B(M)}{B^{3}}\right)\left(\frac{B_{1}^{\prime}}{B_{1}}\right) \cdots\left(\frac{B_{i-1}^{\prime}}{B_{i-1}}\right) ; \frac{B_{i}^{\prime}}{B_{i}}\right] \bmod \mathcal{F}_{2}\left(\Sigma_{1}\right) .
\end{aligned}
$$

We shall now restrict the set of generators $\left[T ; E^{\prime} / E\right]$, where $\left(E^{\prime} / E\right)$ is an elementary surgery of genus 0 .

Lemma 5.7 Let $T$ be a framed rational homology torus and let $B$ be a rational homology ball. Then $\left[T ; B / B^{3}\right] \in \bigoplus_{p \text { prime }} \mathbb{Q}\left[T_{0} ; B_{p} / B^{3}\right] \subset \mathcal{G}_{1}\left(\Sigma_{1}\right)$.

This result follows from the next two sublemmas.

Sublemma 5.8 Let $T$ be a framed rational homology torus and let $B$ be a rational homology ball. Then, in $\mathcal{G}_{1}\left(\Sigma_{1}\right),\left[T ; B / B^{3}\right]$ is a linear combination of the $\left[T ; B_{p} / B^{3}\right]$.

Proof Set $M=B \bigcup_{\partial B=-\partial B^{3}} B^{3}$. We have $T\left(B / B^{3}\right)=T \sharp M$ and $T\left(B_{p} / B^{3}\right)=$ $T \sharp M_{p}$. Now use that $\left(M_{p}-S^{3}\right)_{p}$ prime generates $\mathcal{G}_{1}$ (see Section 4.1).

Sublemma 5.9 For any framed rational homology torus $T$, and any rational homology ball $B$,

$$
\left[T ; \frac{B}{B^{3}}\right]=\left[T_{0} ; \frac{B}{B^{3}}\right] \bmod \mathcal{F}_{2}\left(\Sigma_{1}\right) .
$$

Proof Define $T_{0}^{\prime}$ as $T_{0}$ minus a regular open neighborhood of its boundary. We can suppose that $T_{0}^{\prime}$ and $B^{3}$ are disjoint in $T_{0}$. We have $T \cong T_{0}\left(T / T_{0}^{\prime}\right)$, and

$$
\left[T_{0} ; \frac{T}{T_{0}^{\prime}}, \frac{B}{B^{3}}\right]=\left[T_{0} ; \frac{B}{B^{3}}\right]-\left[T ; \frac{B}{B^{3}}\right] .
$$


Proof of Proposition 5.1 By Lemmas 5.6 and 5.7, the quotient $\mathcal{G}_{1}\left(\Sigma_{1}\right)$ is generated by the $\left[T_{0} ; B_{p} / B^{3}\right]$ and the $\left[T ; A^{\prime} / A\right]$ where $\left(A^{\prime} / A\right)$ is a Borromean surgery. Consider $\mu \in\left(\mathcal{G}_{1}\left(\Sigma_{1}\right)\right)^{*}$. For all prime integer $p$, set $c_{p}=\mu\left(\left[T_{0} ; B_{p} / B^{3}\right]\right)$. Set $\tilde{\mu}=\mu-$ $\sum_{p \text { prime }} c_{p} \mu_{p}$. The invariant $\tilde{\mu}$ is determined by its values on the terms $\left[T ; A^{\prime} / A\right]$, where $\left(A^{\prime} / A\right)$ is a Borromean surgery. Let $\Gamma$ denote the Y-graph associated with the Borromean surgery $\left(A^{\prime} / A\right)$. By Lemma 3.13 , if $T$ is fixed, $\tilde{\mu}\left(\left[T ; A^{\prime} / A\right]\right)$ only depends on the rational homology classes of the three leaves of $\Gamma$, and this dependance is trilinear and alternating. Since $H_{1}(T ; \mathbb{Q}) \cong \mathbb{Q}$, we have $\tilde{\mu}=0$. Hence $\mu=\sum_{p \text { prime }} c_{p} \mu_{p}$. This implies that $\mathcal{G}_{1}\left(\Sigma_{1}\right)$ is generated by the $\left[T_{0} ; B_{p} / B^{3}\right]$. Conclude with Corollary 5.3.

Corollary 5.10 If $\mu$ is a degree 1 invariant of framed rational homology tori, such that $\mu\left(T_{0}\right)=0$ and $\mu\left(T_{0} \sharp M_{p}\right)=0$ for any prime $p$, then $\mu=0$.

\subsection{The quotients $\mathcal{I}_{n}^{c} / \mathcal{I}_{n-1}^{c}$}

The main point of this subsection will be the proof of the next proposition. We will end the subsection by showing that this result implies Proposition 1.11.

Proposition 5.11 If $\lambda$ is an additive invariant of degree $n>1$, then $\lambda_{\mid \mathcal{F}_{n}}$ is determined by $\lambda\left(\Phi\left(\mathcal{A}_{n / 2}^{c}\right)\right)$. In particular, if $n$ is odd, $\lambda_{\mid \mathcal{F}_{n}}=0$.

Recall the map $\Phi: \mathcal{A}_{n} \rightarrow \mathcal{G}_{2 n}^{\mathbb{Z}}$ has been defined in Lemma 1.2. Since we have a canonical map $\mathcal{G}_{2 n}^{\mathbb{Z}} \rightarrow \mathcal{G}_{2 n}$ (we will see later that it is an embedding), $\lambda\left(\Phi\left(\mathcal{A}_{n}^{c}\right)\right.$ ) is well defined.

We will often use the following easy formula.

Lemma 5.12 For any $\left[M ;\left(A_{i}^{\prime} / A_{i}\right)_{1 \leq i \leq n}\right] \in \mathcal{F}_{n}$,

$$
\left[M ;\left(\frac{A_{i}^{\prime}}{A_{i}}\right)_{1 \leq i \leq n}\right]=\left[M ;\left(\frac{A_{i}^{\prime}}{A_{i}}\right)_{2 \leq i \leq n}\right]-\left[M\left(\frac{A_{1}^{\prime}}{A_{1}}\right) ;\left(\frac{A_{i}^{\prime}}{A_{i}}\right)_{2 \leq i \leq n}\right]
$$

Lemma 5.13 The space $\mathcal{G}_{n}$ is generated by the $\left[M ;\left(E_{i}^{\prime} / E_{i}\right)_{1 \leq i \leq n}\right]$, where the $\left(E_{i}^{\prime} / E_{i}\right)$ are elementary surgeries.

To see this, just adapt the proof of Lemma 5.4.

Lemma 5.14 Let $\lambda$ be an additive invariant of degree $n>1$. Let $\left[M ;\left(E_{i}^{\prime} / E_{i}\right)_{1 \leq i \leq n}\right] \in$ $\mathcal{F}_{n}$. If at least one of the surgeries $\left(E_{i}^{\prime} / E_{i}\right)$ is an elementary surgery of genus 0 (connected sum), then $\lambda\left(\left[M ;\left(E_{i}^{\prime} / E_{i}\right)_{1 \leq i \leq n}\right]\right)=0$. 
Proof Assume $E_{1}^{\prime} / E_{1}$ is a connected sum, ie, $E_{1}$ is a ball $B^{3}$, and $E_{1}^{\prime}$ is a rational homology ball. Define a $\mathbb{Q} H S M_{1}$ by gluing $E_{1}^{\prime}$ and a ball $B^{3}$ along their boundaries. Then

$$
\begin{aligned}
\lambda\left(\left[M ;\left(\frac{E_{i}^{\prime}}{E_{i}}\right)_{1 \leq i \leq n}\right]\right) & =\lambda\left(\left[M ;\left(\frac{E_{i}^{\prime}}{E_{i}}\right)_{2 \leq i \leq n}\right]\right)-\lambda\left(\left[M \sharp M_{1} ;\left(\frac{E_{i}^{\prime}}{E_{i}}\right)_{2 \leq i \leq n}\right]\right) \\
& =-\sum_{I \subset\{2, \ldots, n\}}(-1)^{|I|} \lambda\left(M_{1}\right)=0 .
\end{aligned}
$$

Lemma 5.15 Consider a $\mathbb{Q H S} M$, and disjoint LP-surgeries

$$
\left(T_{d} / T_{0}\right), \quad\left(A_{i}^{\prime} / A_{i}\right)_{1 \leq i \leq n-1}
$$

in $M$, where $T_{0}$ is a standard torus, and $T_{d}$ is a $d$-torus. If $\lambda$ is an additive invariant of degree $n>1$, then $\lambda\left(\left[M ; T_{d} / T_{0},\left(A_{i}^{\prime} / A_{i}\right)_{1 \leq i \leq n-1}\right]\right)=0$.

Proof Fix $M$, the embedding of $T_{0}$, and the surgeries $\left(A_{i}^{\prime} / A_{i}\right)_{1 \leq i \leq n-1}$. Fix a longitude $\ell\left(T_{0}\right)$ of $T_{0}$. For any framed rational homology torus $T$, set

$$
\bar{\lambda}(T)=\lambda\left(\left[M ; \frac{T}{T_{0}},\left(\frac{A_{i}^{\prime}}{A_{i}}\right)_{1 \leq i \leq n-1}\right]\right) .
$$

Then $\bar{\lambda}$ is a degree 1 invariant of framed rational homology tori:

$$
\bar{\lambda}\left(\left[T ; \frac{B_{1}^{\prime}}{B_{1}}, \frac{B_{2}^{\prime}}{B_{2}}\right]\right)=\lambda\left(-\left[M\left(\frac{T}{T_{0}}\right) ; \frac{B_{1}^{\prime}}{B_{1}}, \frac{B_{2}^{\prime}}{B_{2}},\left(\frac{A_{i}^{\prime}}{A_{i}}\right)_{1 \leq i \leq n-1}\right]\right)=0 .
$$

We have $\bar{\lambda}\left(T_{0}\right)=\lambda(0)=0$, and

$$
\bar{\lambda}\left(T_{0} \sharp M_{p}\right)=\lambda\left(\left[M ; \frac{B_{p}}{B^{3}},\left(\frac{A_{i}^{\prime}}{A_{i}}\right)_{1 \leq i \leq n-1}\right]\right)=0,
$$

since $\lambda$ is additive, and $n-1>0$. By Corollary $5.10, \bar{\lambda}=0$.

Proof of Proposition 5.11 By Lemmas 5.14 and 5.15, an additive invariant $\lambda$ of degree $n>1$ is determined on $\mathcal{F}_{n}$ by its values on the $\left[M ;\left(B_{i}^{\prime} / B_{i}\right)_{1 \leq i \leq n}\right]$, for all $\mathbb{Q} H S s \quad M$ and all sets of $n$ disjoint Borromean surgeries $\left(B_{i}^{\prime} / B_{i}\right)_{1 \leq i \leq n}$ in $M$. Hence, by Corollary $3.10, \lambda$ is determined on $\mathcal{F}_{n}$ by the $\lambda([M ; \Gamma])$ for all $\mathbb{Q H S} M$ and all Jacobi diagram $\Gamma$ of degree $n / 2$.

We can write $M=M \sharp S^{3}$ and suppose $\Gamma$ is embedded in $S^{3}$. Hence for an additive invariant $\lambda$ of degree $n$, we have $\lambda([M ; \Gamma])=\lambda\left(\left[S^{3} ; \Gamma\right]\right)$.

If the Jacobi diagram $\Gamma$ is not connected, we can assume that $\Gamma$ is made of two components $\Gamma_{1}$ and $\Gamma_{2}$ that are embedded in disjoint balls in $S^{3}$. Noting that $\left(S^{3}, \Gamma\right)=$ 
$\left(S^{3}, \Gamma_{1}\right) \sharp\left(S^{3}, \Gamma_{2}\right)$, it is easy to see that any additive invariant vanishes on $\left[S^{3} ; \Gamma\right]$ in this case.

Proposition 1.11 follows from Proposition 5.11 in the case of odd degrees. For even degrees, it is a consequence of the following lemma.

Lemma 5.16 Let $n>1$ be an even integer. Let $\left(\Gamma_{n, i}\right)_{i \in C_{n}}$ be a basis of diagrams of the finite dimensional vector space $\mathcal{A}_{n / 2}^{c}$. Let $\left(\Gamma_{n, i}^{*}\right)_{i \in C_{n}}$ be the dual basis of $\left(\mathcal{A}_{n / 2}^{c}\right)^{*}$. Let $Z_{n / 2}$ denote the degree $n / 2$ part of the KKT invariant. Let $p^{c}: \mathcal{A}_{n / 2} \rightarrow \mathcal{A}_{n / 2}^{c}$ be the projection that maps any nonconnected diagram to 0 and which restricts to the identity on $\mathcal{A}_{n / 2}^{c}$. For $i \in C_{n}$, set $\lambda_{n, i}=\Gamma_{n, i}^{*} \circ p^{c} \circ Z_{n / 2}$. Then $\left(\lambda_{n, i}\right)_{i \in C_{n}}$ is a basis of $\mathcal{I}_{n}^{c} / \mathcal{I}_{n-1}^{c}$.

Proof By [7, Theorem 1], $p^{c} \circ Z_{n / 2}$ is an additive invariant of $\mathbb{Q} H S s$, thus the $\lambda_{n, i}$ are additive. By [10, Theorem 2.4] and [1, Proposition 4.1], $Z_{n / 2}$ is a finite type invariant of degree $\mathrm{n}$ and satisfies $Z_{n / 2}\left(\left[S^{3} ; \Gamma_{n, i}\right]\right)=\Gamma_{n, i} \in \mathcal{A}_{n / 2}$. Hence $\lambda_{n, i} \in \mathcal{I}_{n}^{c}$, and $\lambda_{n, i}\left(\left[S^{3} ; \Gamma_{n, j}\right]\right)=\delta_{i, j}$. Consider $\lambda \in \mathcal{I}_{n}^{c}$. By Proposition 5.11,

$$
\lambda=\sum_{i \in C_{n}} \lambda\left(\left[S^{3} ; \Gamma_{n, i}\right]\right) \lambda_{n, i}
$$

in $\mathcal{I}_{n}^{c} / \mathcal{I}_{n-1}^{c}$. Hence $\left(\lambda_{n, i}\right)_{i \in C_{n}}$ is a basis of $\mathcal{I}_{n}^{c} / \mathcal{I}_{n-1}^{c}$.

\section{The graded algebras $\mathcal{G}$ and $\mathcal{H}$}

\subsection{The products in $\mathcal{G}$ and $\mathcal{H}$}

Extend the connected sum to $\mathcal{F}_{0}$ by bilinearity:

$$
\left(\sum_{i \in I} a_{i} M_{i}\right) \sharp\left(\sum_{j \in J} b_{j} N_{j}\right)=\sum_{i \in I} \sum_{j \in J} a_{i} b_{j}\left(M_{i} \sharp N_{j}\right),
$$

for any finite sets $I$ and $J$, any rational numbers $a_{i}, b_{j}$, and any $\mathbb{Q}$ HSs $M_{i}, N_{j}$.

Lemma 6.1 $\mathcal{F}_{n} \sharp \mathcal{F}_{m} \subset \mathcal{F}_{n+m}$.

Proof Just check that

$\left[M ;\left(\frac{B_{i}}{A_{i}}\right)_{1 \leq i \leq n}\right] \sharp\left[M^{\prime} ;\left(\frac{B_{i}^{\prime}}{A_{i}^{\prime}}\right)_{1 \leq i \leq m}\right]=\left[M \sharp M^{\prime} ;\left(\frac{B_{i}}{A_{i}}\right)_{1 \leq i \leq n},\left(\frac{B_{i}^{\prime}}{A_{i}^{\prime}}\right)_{1 \leq i \leq m}\right] \square$ 
Thus the connected sum defines a product $\sharp: \mathcal{G}_{n} \times \mathcal{G}_{m} \rightarrow \mathcal{G}_{n+m}$ which induces a graded algebra structure on $\mathcal{G}$.

Given two finite type invariants $\lambda$ and $\mu$, note that the product $\lambda \mu$ satisfies

$$
\lambda \mu\left(\sum_{i \in I} a_{i} M_{i}\right)=\sum_{i \in I} a_{i} \lambda\left(M_{i}\right) \mu\left(M_{i}\right)
$$

for any finite set $I$, rational numbers $a_{i}$, and $\mathbb{Q H S s} M_{i}$.

Lemma 6.2 If $\lambda \in \mathcal{I}_{k}$ and $\mu \in \mathcal{I}_{\ell}$, then $\lambda \mu \in \mathcal{I}_{k+\ell}$.

Proof Consider $\left[M ;\left(B_{i} / A_{i}\right)_{i \in I}\right]$ with $|I|=k+\ell+1$. We have the following equality:

(1) $\lambda \mu\left(\left[M ;\left(\frac{B_{i}}{A_{i}}\right)_{i \in I}\right]\right)$

$$
=\sum_{J \subset I} \lambda\left(\left[M ;\left(\frac{B_{i}}{A_{i}}\right)_{i \in J}\right]\right) \mu\left(\left[M\left(\left(\frac{B_{i}}{A_{i}}\right)_{i \in J}\right) ;\left(\frac{B_{i}}{A_{i}}\right)_{i \in I \backslash J}\right]\right) .
$$

Indeed, the right hand side is equal to

$$
\begin{aligned}
& \sum_{J \subset I}(-1)^{|J|}\left(\sum_{K \subset J}(-1)^{|K|} \lambda\left(M\left(\left(\frac{B_{i}}{A_{i}}\right)_{i \in K}\right)\right)\right)\left(\sum_{L \supset J}(-1)^{|L|} \mu\left(M\left(\left(\frac{B_{i}}{A_{i}}\right)_{i \in L}\right)\right)\right) \\
& =\sum_{L \subset I} \sum_{K \subset L}(-1)^{|K|+|L|} \lambda\left(M\left(\left(\frac{B_{i}}{A_{i}}\right)_{i \in K}\right)\right) \mu\left(M\left(\left(\frac{B_{i}}{A_{i}}\right)_{i \in L}\right)\right)\left(\sum_{K \subset J \subset L}(-1)^{|J|}\right) .
\end{aligned}
$$

Since

$$
\sum_{K \subset J \subset L}(-1)^{|J|}= \begin{cases}0 & \text { if } K \subsetneq L \\ (-1)^{|K|} & \text { if } K=L\end{cases}
$$

we get (1).

In (1), we have, if $|J|>k$,

$$
\lambda\left(\left[M ;\left(\frac{B_{i}}{A_{i}}\right)_{i \in J}\right]\right)=0,
$$

and, if $|J| \leq k$, then $|I \backslash J|>\ell$ and

$$
\mu\left(\left[M\left(\left(\frac{B_{i}}{A_{i}}\right)_{i \in J}\right) ;\left(\frac{B_{i}}{A_{i}}\right)_{i \in I \backslash J}\right]\right)=0 .
$$

Thus

$$
\lambda \mu\left(\left[M ;\left(\frac{B_{i}}{A_{i}}\right)_{i \in I}\right]\right)=0
$$


Thus the product of finite type invariants induces a graded algebra structure on $\mathcal{H}$.

\subsection{Dual systems in $\mathcal{G}$ and $\mathcal{H}$}

For an even integer $n>1$, consider the basis $\left(\Gamma_{n, i}\right)_{i \in C_{n}}$ of $\mathcal{A}_{n / 2}^{c}$ and the associated invariants $\lambda_{n, i}$ defined in Lemma 5.16. For $n>1$ odd, set $C_{n}=\varnothing$. For $n=1$, let $C_{1}$ denote the set of all prime integers, and for any $p$ prime, set $\lambda_{1, p}=v_{p}$ and $\Gamma_{1, p}=\bullet{ }_{p} \in \mathcal{A}_{1}^{\text {aug }}$. Note that adding to $\lambda_{n, i}$ a weighted sum of the $\lambda_{k, i}, 0<k<n$, $i \in C_{k}$, does not change the values of $\lambda_{n, i}$ on $\mathcal{F}_{n}$. Thus we can (and we do) choose the basis $\left(\lambda_{n, i}\right)_{i \in C_{n}}$ so that $\lambda_{n, i}\left(\left[S^{3} ; \Gamma_{k, j}\right]\right)=\delta_{n k} \delta_{i j}$ for all positive integers $n$ and $k$, all $i \in C_{n}$, all $j \in C_{k}$.

For a multiindex $\underline{\varepsilon}=\left(\varepsilon_{t}\right)_{1 \leq t \leq \ell}$, set $\ell(\underline{\varepsilon})=\ell$. For $n>0$, fix a total order on $C_{n}$. Let $\preccurlyeq$ denote the lexicographic order induced on $\bigcup_{n \in \mathbb{N} \backslash\{0\}}\left(\{n\} \times C_{n}\right)$. For $n>0$, let $\mathcal{T}_{n}^{\pi}$ denote the set of all triples $(\underline{k}, \underline{i}, \underline{\varepsilon})$ such that $\ell(\underline{k})=\ell(\underline{i})=\ell(\underline{\varepsilon}), \underline{k}=\left(k_{t}\right)_{1 \leq t \leq \ell(\underline{k})}$, $k_{t} \in \mathbb{N}$ and $0<k_{t}<n$ for all $t, \underline{i}=\left(i_{t}\right)_{1 \leq t \leq \ell(k)}$ with $i_{t} \in C_{k_{t}}$ for all $t,\left(k_{1}, i_{1}\right) \prec$ $\left(k_{2}, i_{2}\right) \prec \cdots \prec\left(k_{\ell(\underline{k})}, i_{\ell(k)}\right), \underline{\varepsilon}=\left(\varepsilon_{t}\right)_{1 \leq t \leq \ell(\varepsilon)}$ with $\varepsilon_{t} \in \mathbb{N} \backslash\{0\}$ for all $t$, and $\sum_{1 \leq t \leq \ell(k)} \varepsilon_{t} k_{t}=n$. Define a family $\left(\lambda_{n, l}\right)_{\iota \in \mathcal{T}_{n}^{\pi}}$ of invariants of degree $n$ by

$$
\lambda_{n, l}=\prod_{1 \leq t \leq \ell(\underline{k})} \lambda_{k_{t}, i_{t}}^{\varepsilon_{t}},
$$

if $\iota=(\underline{k}, \underline{i}, \underline{\varepsilon})$. Set $\mathcal{T}_{n}=C_{n} \sqcup \mathcal{T}_{n}^{\pi}$. We will see in Section 6.3 that the family $\left(\lambda_{k, i}\right)_{0<k \leq n, i \in \mathcal{T}_{k}}$ is a basis of $\mathcal{I}_{n} / \mathcal{I}_{0}$. The main goal of this subsection is to construct a family $\left(G_{k, i}^{(n)}\right)_{0<k \leq n, i \in \mathcal{T}_{k}}$ of $\mathcal{F}_{1} / \mathcal{F}_{n+1}$, dual to $\left(\lambda_{k, i}\right)_{0<k \leq n, i \in \mathcal{T}_{k}}$.

Definition 6.3 $G \in \mathcal{F}_{0}$ is said to be multiplicative if $\lambda \mu(G)=\lambda(G) \mu(G)$ for all finite type invariants $\lambda$ and $\mu$ such that $\lambda\left(S^{3}\right)=0$ and $\mu\left(S^{3}\right)=0$.

For any $p$ prime, set $G_{1, p}^{(1)}=M_{p}-S^{3}$. Note that the $G_{1, p}^{(1)}$ are multiplicative. Fix $n>1$. If $n$ is even, set $G_{n, i}^{(n)}=\left[S^{3} ; \Gamma_{n, i}\right]$ for $i \in C_{n}$. Since $\left[S^{3} ; \Gamma_{n, i}\right]=S^{3}\left(\Gamma_{n, i}\right)-S^{3}$, $G_{n, i}^{(n)}$ is multiplicative for all $i \in C_{n}$. For $\iota=(\underline{k}, \underline{i}, \underline{\varepsilon}) \in \mathcal{T}_{n}^{\pi}$, set

$$
\widetilde{G}_{n, l}^{(n)}=\underset{1 \leq t \leq \ell(\underline{k})}{\#}\left(G_{k_{t}, i_{t}}^{\left(k_{t}\right)}\right)^{\# \varepsilon_{t}} .
$$

Lemma 6.4 Consider positive integers $p$ and $q$, additive invariants $\lambda_{1}, \ldots, \lambda_{p}$, and elements $\left[M_{1} ;\left(B_{u} / A_{u}\right)_{u \in U_{1}}\right], \ldots,\left[M_{q} ;\left(B_{u} / A_{u}\right)_{u \in U_{q}}\right]$ of $\mathcal{F}_{0}$, for nonempty sets $U_{j}$. Then

$\left(\prod_{i=1}^{p} \lambda_{i}\right)\left(\underset{j=1}{\sharp}\left[M_{j} ;\left(\frac{B_{u}}{A_{u}}\right)_{u \in U_{j}}\right]\right)=\sum_{j \in E_{p q}} \prod_{\ell=1}^{q}\left(\prod_{i \in j^{-1}(\{\ell\})} \lambda_{i}\right)\left(\left[M_{\ell} ;\left(\frac{B_{u}}{A_{u}}\right)_{u \in U_{\ell}}\right]\right)$, 
where $E_{p q}$ is the set of all surjective maps $j:\{1, \ldots, p\} \rightarrow\{1, \ldots, q\}$.

In particular, if $p<q$,

$$
\left(\prod_{i=1}^{p} \lambda_{i}\right)\left(\underset{j=1}{\sharp}\left[M_{j} ;\left(\frac{B_{u}}{A_{u}}\right)_{u \in U_{j}}\right]\right)=0
$$

and, if $p=q$,

$$
\left(\prod_{i=1}^{p} \lambda_{i}\right)\left(\underset{j=1}{\sharp}\left[M_{j} ;\left(\frac{B_{u}}{A_{u}}\right)_{u \in U_{j}}\right]\right)=\sum_{\sigma \in \mathcal{S}_{p}} \prod_{\ell=1}^{p} \lambda_{\sigma(\ell)}\left(\left[M_{\ell} ;\left(\frac{B_{u}}{A_{u}}\right)_{u \in U_{\ell}}\right]\right)
$$

where $\mathcal{S}_{p}$ is the set of permutations of $\{1, \ldots, p\}$.

\section{Proof}

$$
\begin{aligned}
& \left(\prod_{i=1}^{p} \lambda_{i}\right)\left(\underset{j=1}{\sharp}\left[M_{j} ;\left(\frac{B_{u}}{A_{u}}\right)_{u \in U_{j}}\right]\right) \\
& =\sum_{V_{1} \subset U_{1}} \cdots \sum_{V_{q} \subset U_{q}}(-1)^{\sum_{\ell=1}^{q}\left|V_{\ell}\right|} \prod_{i=1}^{p} \lambda_{i}\left(\underset{j=1}{\sharp} M_{j}\left(\left(\frac{B_{u}}{A_{u}}\right)_{u \in V_{j}}\right)\right) \\
& =\sum_{V_{1} \subset U_{1}} \cdots \sum_{V_{q} \subset U_{q}}(-1)^{\sum_{\ell=1}^{q}\left|V_{\ell}\right|} \prod_{i=1}^{p} \sum_{j=1}^{q} \lambda_{i}\left(M_{j}\left(\left(\frac{B_{u}}{A_{u}}\right)_{u \in V_{j}}\right)\right) \\
& =\sum_{j:\{1, \ldots, p\} \rightarrow\{1, \ldots, q\}} \sum_{V_{1} \subset U_{1}} \ldots \sum_{V_{q} \subset U_{q}}(-1)^{\sum_{\ell=1}^{q}\left|V_{\ell}\right|} \prod_{i=1}^{p} \lambda_{i}\left(M_{j(i)}\left(\left(\frac{B_{u}}{A_{u}}\right)_{u \in V_{j(i)}}\right)\right) \\
& =\sum_{j:\{1, \ldots, p\} \rightarrow\{1, \ldots, q\}} \prod_{\ell=1}^{q}\left(\sum_{V_{\ell} \subset U_{\ell}}(-1)^{\left|V_{\ell}\right|} \prod_{i \in j^{-1}(\{\ell\})} \lambda_{i}\left(M_{\ell}\left(\left(\frac{B_{u}}{A_{u}}\right)_{u \in V_{\ell}}\right)\right)\right) \\
& =\sum_{j \in E_{p q}} \prod_{\ell=1}^{q}\left(\prod_{i \in j^{-1}(\{\ell\})} \lambda_{i}\right)\left(\left[M_{\ell} ;\left(\frac{B_{u}}{A_{u}}\right)_{u \in U_{\ell}}\right]\right)
\end{aligned}
$$

Lemma 6.5 Let $n$ and $k$ be positive integers. For $\iota=(\underline{k}, \underline{i}, \underline{\varepsilon}) \in \mathcal{T}_{k}^{\pi}$, set $\mathcal{T}_{n}(\iota)=$ $\left\{(\underline{k}, \underline{i}, \underline{\eta}) \in \mathcal{T}_{n}^{\pi} \mid \forall t, \eta_{t} \geq \varepsilon_{t}\right\}$. For $\kappa \in \mathcal{T}_{n}^{\pi}$, we have

$$
\lambda_{n, \kappa}\left(\widetilde{G}_{k, l}^{(k)}\right) \neq 0 \quad \text { if and only if } \quad \kappa \in \mathcal{T}_{n}(\iota)
$$

Note that the set $\mathcal{T}_{n}(\iota)$ is finite. 
Proof Set $\kappa=(\underline{\ell}, \underline{j}, \underline{\eta})$. We have

$$
\lambda_{n, \kappa}=\prod_{1 \leq s \leq \ell(\underline{\ell})} \lambda_{\ell_{s}, j_{s}}^{\eta_{s}}, \quad \widetilde{G}_{k, \iota}^{(k)}=\prod_{1 \leq t \leq \ell(\underline{k})}^{\sharp}\left(G_{k_{t}, i_{t}}^{\left(k_{t}\right)}\right)^{\sharp \varepsilon_{t}} .
$$

By Lemma 6.4, we have

$$
\lambda_{n, \kappa}\left(\widetilde{G}_{k, l}^{(k)}\right)=\sum_{\xi \in E_{\underline{n} \underline{\varepsilon}}} \prod_{(t, u) \in \Theta(\underline{\varepsilon})}\left(\prod_{(s, v) \in \xi^{-1}(\{(t, u)\})} \lambda_{\ell_{s}, j_{s}}\right)\left(G_{k_{t}, i_{t}}^{\left(k_{t}\right)}\right),
$$

where $\Theta(\underline{\varepsilon})=\left\{(t, u) \mid 1 \leq t \leq \ell(\underline{\varepsilon}) ; \forall t, 1 \leq u \leq \varepsilon_{t}\right\}$ and $E_{\eta \underline{\varepsilon}}$ is the set of all surjective maps $\xi: \Theta(\underline{\eta}) \rightarrow \Theta(\underline{\varepsilon})$. Since the $G_{k_{t}, i_{t}}^{\left(k_{t}\right)}$ are multiplicative, we get

$$
\lambda_{n, \kappa}\left(\widetilde{G}_{k, l}^{(k)}\right)=\sum_{\xi \in E_{\underline{\eta} \underline{\varepsilon}}} \prod_{(t, u) \in \Theta(\underline{\varepsilon})} \prod_{(s, v) \in \xi^{-1}(\{(t, u)\})}\left(\lambda_{\ell_{s}, j_{s}}\left(G_{k_{t}, i_{t}}^{\left(k_{t}\right)}\right)\right) .
$$

Recall that $\lambda_{\ell_{s}, j_{s}}\left(G_{k_{t}, i_{t}}^{\left(k_{t}\right)}\right)=\delta_{\ell_{s} k_{t}} \delta_{j_{s} i_{t}}$. Hence $\lambda_{n, \kappa}\left(\widetilde{G}_{k, l}^{(k)}\right) \neq 0$ if and only if $\underline{\ell}=\underline{k}$, $\underline{j}=\underline{i}$ and $\eta_{t} \geq \varepsilon_{t}$ for all $t$.

For $n>1$ and $\iota \in \mathcal{T}_{n}^{\pi}$, set $G_{n, l}^{(n)}=\frac{1}{\lambda_{n, l}\left(\widetilde{G}_{n, l}^{(n)}\right)} \widetilde{G}_{n, l}^{(n)}$, so that $\lambda_{n, l}\left(G_{n, l}^{(n)}\right)=1$. Note that, for all $n$ and all $i \in \mathcal{T}_{n}, G_{n, i}^{(n)} \in \mathcal{F}_{n}{ }^{\lambda}$.

Let $n$ and $k$ be positive integers. For $\iota \in C_{k}$, set

$$
\mathcal{T}_{n}(\iota)=\left\{(\underline{k}, \underline{i}, \underline{\eta}) \in \mathcal{T}_{n}^{\pi} \mid \underline{k}=(k), \underline{i}=(\iota)\right\} .
$$

The following result is an easy generalization of Lemma 6.5.

Lemma 6.6 Let $n$ and $k$ be positive integers. For $\kappa \in \mathcal{T}_{n}$ and $\iota \in \mathcal{T}_{k}$, we have $\lambda_{n, \kappa}\left(G_{k, \iota}^{(k)}\right) \neq 0$ if and only if $\kappa \in \mathcal{T}_{n}(\iota)$.

Corollary 6.7 For $n>0, i \in \mathcal{T}_{n}, j \in \mathcal{T}_{n}$, we have $\lambda_{n, i}\left(G_{n, j}^{(n)}\right)=\delta_{i j}$.

For $n>1$, define $G_{k, i}^{(n)} \in \mathcal{F}_{k}$ for $0<k<n$ and $i \in \mathcal{T}_{k}$, by induction on $n$, by

$$
G_{k, i}^{(n)}=G_{k, i}^{(n-1)}-\sum_{\iota \in \mathcal{T}_{n}(i)} \lambda_{n, \iota}\left(G_{k, i}^{(n-1)}\right) G_{n, \iota}^{(n)} .
$$

Note that $G_{k, i}^{(n)}=G_{k, i}^{(m)}$ in $\mathcal{G}_{m}$ if $m \leq n$.

Lemma 6.8 Let $n$ be a positive integer. The family $\left(G_{k, i}^{(n)}\right)_{0<k \leq n, i \in \mathcal{T}_{k}}$ of $\mathcal{F}_{1} / \mathcal{F}_{n+1}$ is dual to the family $\left(\lambda_{k, i}\right)_{0<k \leq n, i \in \mathcal{T}_{k}}$ of $\mathcal{I}_{n} / \mathcal{I}_{0}$. 
Proof We proceed by induction on $n$. The result is clear for $n=1$. Fix $n>1$. We shall prove that $\lambda_{\ell, j}\left(G_{k, i}^{(n)}\right)=\delta_{\ell k} \delta_{j i}$ for all $0<\ell \leq n, j \in \mathcal{T}_{\ell}, 0<k \leq n, i \in \mathcal{T}_{k}$. If $\ell=n$ and $k=n$, it is given by Corollary 6.7. If $\ell<n$ and $k=n$, it is clear since $G_{n, i}^{(n)} \in \mathcal{F}_{n}$. If $\ell<n$ and $k<n$, it follows from the induction hypothesis. It remains to show that $\lambda_{n, j}\left(G_{k, i}^{(n)}\right)=0$ if $k<n$. It is immediate if $j \in \mathcal{T}_{n}(i)$. Consider $j \in \mathcal{T}_{n} \backslash \mathcal{T}_{n}(i)$. We have

$$
G_{k, i}^{(n)}=G_{k, i}^{(k)}-\sum_{k<m \leq n} \sum_{n \in \mathcal{T}_{m}(i)} \lambda_{m, l}\left(G_{k, i}^{(m-1)}\right) G_{m, \iota}^{(m)}
$$

By Lemma 6.6, for $k \leq m \leq n$ and $\iota \in \mathcal{T}_{m}(i), \lambda_{n, j}\left(G_{m, \iota}^{(m)}\right) \neq 0$ if and only if $j \in \mathcal{T}_{n}(\iota)$, and this implies $j \in \overline{\mathcal{T}}_{n}(i)$. Hence, for $j \notin \mathcal{T}_{n}(i), \lambda_{n, j}\left(G_{k, i}^{(n)}\right)=0$.

\subsection{The coproduct on $\mathcal{H}$}

In the previous subsection, we have constructed dual systems

$$
\left(G_{k, i}^{(n)}\right)_{0<k \leq n, i \in \mathcal{T}_{k}} \subset \frac{\mathcal{F}_{1}}{\mathcal{F}_{n+1}}, \quad\left(\lambda_{k, i}\right)_{0<k \leq n, i \in \mathcal{T}_{k}} \subset \frac{\mathcal{I}_{n}}{\mathcal{I}_{0}},
$$

that satisfy the following properties:

- $\lambda_{n, i}$ is a finite type invariant of degree $n$;

- $\mathcal{T}_{n}=C_{n} \sqcup \mathcal{T}_{n}^{\pi}, \lambda_{n, i}$ is additive if $i \in C_{n}, \lambda_{n, i}$ is a product of some $\lambda_{k, i}, k<n$, $i \in C_{k}$, if $i \in \mathcal{T}_{n}^{\pi}$;

- $\mathcal{I}_{n}^{c} / \mathcal{I}_{n-1}^{c}=\prod_{i \in C_{n}} \mathbb{Q} \lambda_{n, i}$;

- if $i \in C_{n}, G_{n, i}^{(n)}$ is multiplicative;

- $G_{k, i}^{(n)} \in \mathcal{F}_{k}$, and, if $m \leq n, G_{k, i}^{(n)}=G_{k, i}^{(m)}$ in $\mathcal{G}_{m}$.

Proposition 6.9 The family $\left(G_{k, i}^{(n)}\right)_{0<k \leq n, i \in \mathcal{T}_{k}}$ is a basis of $\mathcal{F}_{1} / \mathcal{F}_{n+1}$. The family $\left(\lambda_{k, i}\right)_{0<k \leq n, i \in \mathcal{T}_{k}}$ is the dual basis of $\mathcal{I}_{n} / \mathcal{I}_{0}$. Moreover,

$$
\frac{\mathcal{I}_{n}}{\mathcal{I}_{n-1}}=\prod_{i \in \mathcal{T}_{n}} \mathbb{Q} \lambda_{n, i}, \quad \frac{\mathcal{I}_{n}^{\pi}}{\mathcal{I}_{n-1}^{\pi}}=\prod_{i \in \mathcal{T}_{n}^{\pi}} \mathbb{Q} \lambda_{n, i}, \quad \mathcal{G}_{n}=\bigoplus_{i \in \mathcal{T}_{n}} \mathbb{Q} G_{n, i}^{(n)} .
$$

This result implies Proposition 1.12.

Proof We will proceed by induction. For $n=1$, the result follows from Proposition 1.8 and Corollary 1.10. Fix $n>1$. We will write $\underline{\eta} \leq \underline{\varepsilon}$ if $\eta_{t} \leq \varepsilon_{t}$ for all $t, \underline{\eta}<\underline{\varepsilon}$ if $\underline{\eta} \leq \underline{\varepsilon}$ and $\underline{\eta} \neq \underline{\varepsilon}$, and $\underline{0}<\underline{\eta}$ if $\eta_{t}>0$ for at least one $t$. 
Lemma 6.10 Consider $\lambda \in \mathcal{I}_{n}$ such that $\lambda\left(S^{3}\right)=0$. There are constants $\alpha_{m, \iota}$, for $1 \leq m \leq n$ and $\iota \in \mathcal{T}_{m}^{\pi}$, such that

$$
\begin{aligned}
\lambda\left(M_{1} \sharp M_{2}\right)= & \lambda\left(M_{1}\right)+\lambda\left(M_{2}\right) \\
& +\sum_{m=1}^{n} \sum_{\iota=\left(\underline{k}, \underline{\underline{\varepsilon}}, \underline{\varepsilon} \in \mathcal{T}_{m}^{\pi}\right.} \alpha_{m, \iota} \sum_{\underline{0}<\underline{\eta}<\underline{\varepsilon}} \prod_{1 \leq t \leq \ell(\underline{k})}\left(\begin{array}{l}
\varepsilon_{t} \\
\eta_{t}
\end{array}\right) \lambda_{k_{t}, i_{t}}^{\eta_{t}}\left(M_{1}\right) \lambda_{k_{t}, i_{t}}^{\varepsilon_{t}-\eta_{t}}\left(M_{2}\right),
\end{aligned}
$$

for all $\mathbb{Q} H S s M_{1}$ and $M_{2}$.

Remark The above expression of $\lambda\left(M_{1} \sharp M_{2}\right)$ defines a coproduct $\Delta$ on the algebra $\mathcal{H}$ :

$$
\Delta(\lambda)=\lambda \otimes 1+1 \otimes \lambda+\sum_{m=1}^{n} \sum_{\iota=(\underline{k}, \underline{i}, \underline{\varepsilon}) \in \mathcal{T}_{m}^{\pi}} \alpha_{m, \iota} \sum_{\underline{0}<\underline{\eta}<\underline{\varepsilon}} \prod_{1 \leq t \leq \ell(\underline{k})}\left(\begin{array}{c}
\varepsilon_{t} \\
\eta_{t}
\end{array}\right) \lambda_{k_{t}, i_{t}}^{\eta_{t}} \otimes \lambda_{k_{t}, i_{t}}^{\varepsilon_{t}-\eta_{t}} .
$$

Thus $\mathcal{H}$ has a Hopf algebra structure. The primitive elements associated with this coproduct (the invariants $\lambda$ satisfying $\Delta(\lambda)=\lambda \otimes 1+1 \otimes \lambda$ ) are the additive invariants. Milnor and Moore [14] proved that, under conditions, a Hopf algebra is generated as an algebra by its primitive elements. Here, we give an explicit and elementary proof of this result in our setting.

Proof of Lemma 6.10 Define a bilinear map $\mu$ on $\mathcal{F}_{0}$ by

$$
\mu\left(M_{1}, M_{2}\right)=\lambda\left(M_{1} \sharp M_{2}\right)-\lambda\left(M_{1}\right)-\lambda\left(M_{2}\right)
$$

for all $\mathbb{Q H S s} M_{1}$ and $M_{2}$. Fix $M_{2}$, and consider $\left[M ;\left(A_{i}^{\prime} / A_{i}\right)_{1 \leq i \leq n}\right] \in \mathcal{F}_{n}$. We have

$$
\begin{aligned}
\mu\left(\left[M ;\left(\frac{A_{i}^{\prime}}{A_{i}}\right)_{1 \leq i \leq n}\right], M_{2}\right) & \\
= & \sum_{I \subset\{1, \ldots, n\}}(-1)^{|I|} \mu\left(M\left(\left(\frac{A_{i}^{\prime}}{A_{i}}\right)_{i \in I}\right), M_{2}\right) \\
& =\sum_{I \subset\{1, \ldots, n\}}(-1)^{|I|}\left(\lambda\left(M\left(\left(\frac{A_{i}^{\prime}}{A_{i}}\right)_{i \in I}\right) \sharp M_{2}\right)-\lambda\left(M\left(\left(\frac{A_{i}^{\prime}}{A_{i}}\right)_{i \in I}\right)\right)\right) \\
& =-\lambda\left(\left[M ;\left(\frac{A_{i}^{\prime}}{A_{i}}\right)_{1 \leq i \leq n}, \frac{B_{2}}{B^{3}}\right]\right)=0,
\end{aligned}
$$

where $B_{2}$ is a rational homology ball obtained from $M_{2}$ by removing an open ball. Thus $\mu\left(\cdot, M_{2}\right)$ is an invariant of degree at most $n-1$. Note that $\mu\left(S^{3}, M_{2}\right)=0$. By 
induction, $\mathcal{I}_{n-1} / \mathcal{I}_{0}$ is freely generated by the $\lambda_{k, i}$ for $0<k<n$ and $i \in \mathcal{T}_{k}$. Hence we can write

$$
\mu\left(M_{1}, M_{2}\right)=\sum_{0<k<n} \sum_{i \in \mathcal{T}_{k}} \beta_{k, i}\left(M_{2}\right) \lambda_{k, i}\left(M_{1}\right)
$$

Note that the sum may be infinite. We have $\beta_{k, i}\left(M_{2}\right)=\mu\left(G_{k, i}^{(n)}, M_{2}\right)$ and $\beta_{k, i}\left(S^{3}\right)=0$. Extend $\beta_{k, i}$ to $\mathcal{F}_{0}$ by linearity. Consider $\left[M ;\left(A_{i}^{\prime} / A_{i}\right)_{i \in I}\right] \in \mathcal{F}_{n-k+1},|I|=n-k+1$, and set $G_{k, i}^{(n)}=\sum_{u \in U} c_{u}\left[N_{u} ;\left(B_{j}^{\prime} / B_{j}\right)_{j \in J_{u}}\right]$, where the $c_{u}$ are rational numbers and $\left|J_{u}\right|=k$ for all $u$. We have

$$
\begin{aligned}
\beta_{k, i}\left(\left[M ;\left(\frac{A_{i}^{\prime}}{A_{i}}\right)_{i \in I}\right]\right) \\
\quad=\sum_{u \in U} c_{u} \sum_{I^{\prime} \subset I} \sum_{K_{u} \subset J_{u}}(-1)^{\left|I^{\prime}\right|+\left|K_{u}\right|} \mu\left(N_{u}\left(\left(\frac{B_{j}^{\prime}}{B_{j}}\right)_{j \in K_{u}}\right), M\left(\left(\frac{A_{i}^{\prime}}{A_{i}}\right)_{i \in I^{\prime}}\right)\right) \\
=\sum_{u \in U} c_{u} \lambda\left(\left[M \sharp N_{u} ;\left(\frac{A_{i}^{\prime}}{A_{i}}\right)_{i \in I},\left(\frac{B_{j}^{\prime}}{B_{j}}\right)_{j \in J_{u}}\right]\right)=0 .
\end{aligned}
$$

Thus $\beta_{k, i}$ is an invariant of degree at most $n-k$. Using the induction hypothesis, we can decompose the invariants $\beta_{k, i}$ and get

$$
\mu\left(M_{1}, M_{2}\right)=\sum_{m=1}^{n} \sum_{\iota=(\underline{k}, \underline{i}, \underline{\varepsilon}) \in \mathcal{T}_{m}^{\pi}} \sum_{\underline{0}<\underline{\eta}<\underline{\varepsilon}} \alpha_{m, \iota}^{(\underline{\eta})} \prod_{1 \leq t \leq \ell(\underline{k})}\left(\begin{array}{l}
\varepsilon_{t} \\
\eta_{t}
\end{array}\right) \lambda_{k_{t}, i_{t}}^{\eta_{t}}\left(M_{1}\right) \lambda_{k_{t}, i_{t}}^{\varepsilon_{t}-\eta_{t}}\left(M_{2}\right),
$$

where the $\alpha^{(\eta)}{ }_{m, \iota}$ are rational constants. It gives

$$
\begin{aligned}
\lambda\left(M_{1} \sharp M_{2}\right)= & \lambda\left(M_{1}\right)+\lambda\left(M_{2}\right) \\
& +\sum_{m=1}^{n} \sum_{\iota=(\underline{k}, \underline{i}, \underline{\varepsilon}) \in \mathcal{T}_{m}^{\pi}} \sum_{\underline{0}<\underline{\eta}<\underline{\varepsilon}} \alpha_{\frac{\eta}{m, \iota}}^{(\underline{)}} \prod_{1 \leq t \leq \ell(\underline{k})}\left(\begin{array}{l}
\varepsilon_{t} \\
\eta_{t}
\end{array}\right) \lambda_{k_{t}, i_{t}}^{\eta_{t}}\left(M_{1}\right) \lambda_{k_{t}, i_{t}}^{\varepsilon_{t}-\eta_{t}}\left(M_{2}\right) .
\end{aligned}
$$

Now, we use the commutativity and associativity of the connected sum to show that the well-determined constants $\alpha{ }^{(\eta)}{ }_{m, \iota}$ do not depend on $\underline{\eta}$. The commutativity gives 
$\alpha^{(\underline{\varepsilon}-\underline{\eta})}{ }_{m, \iota}=\alpha^{(\underline{\eta})}{ }_{m, l}$. Consider $M_{1}=N_{1} \sharp N_{2}$. We have

$\lambda\left(N_{1} \sharp N_{2} \sharp M_{2}\right)$

$$
\begin{aligned}
& =\lambda\left(N_{1}\right)+\lambda\left(N_{2}\right)+\lambda\left(M_{2}\right) \\
& +\sum_{m=1}^{n} \sum_{\iota=\left(\underline{k}, \underline{i}, \underline{\varepsilon} \in \in \mathcal{T}_{m}^{\pi}\right.} \sum_{\underline{0}<\underline{\eta}<\underline{\varepsilon}} \alpha_{m, \iota}^{(\underline{\eta})} \prod_{1 \leq t \leq \ell(\underline{k})}\left(\begin{array}{l}
\varepsilon_{t} \\
\eta_{t}
\end{array}\right) \lambda_{k_{t}, i_{t}}^{\eta_{t}}\left(N_{1}\right) \lambda_{k_{t}, i_{t}}^{\varepsilon_{t}-\eta_{t}}\left(N_{2}\right) \\
& +\sum_{m=1}^{n} \sum_{\iota=(\underline{k}, \underline{i}, \underline{\varepsilon}) \in \mathcal{T}_{m}^{\pi}} \sum_{\underline{0}<\underline{\eta}<\underline{\varepsilon}} \alpha_{m, \iota}^{(\underline{\eta})} \sum_{\underline{0} \leq \underline{v} \leq \underline{\eta}} \prod_{1 \leq t \leq \ell(k)}\left(\begin{array}{l}
\varepsilon_{t} \\
\eta_{t}
\end{array}\right)\left(\begin{array}{l}
\eta_{t} \\
v_{t}
\end{array}\right) \\
& \times \lambda_{k_{t}, i_{t}}^{v_{t}}\left(N_{1}\right) \lambda_{k_{t}, i_{t}}^{\eta_{t}-v_{t}}\left(N_{2}\right) \lambda_{k_{t}, i_{t}}^{\varepsilon_{t}-\eta_{t}}\left(M_{2}\right) .
\end{aligned}
$$

Consider $\underline{v}$ such that $\underline{0}<\underline{v} \leq \underline{\eta}$. The terms

$$
\begin{gathered}
\prod_{1 \leq t \leq \ell(\underline{k})} \lambda_{k_{t}, i_{t}}^{v_{t}}\left(N_{1}\right) \lambda_{k_{t}, i_{t}}^{\eta_{t}-v_{t}}\left(N_{2}\right) \lambda_{k_{t}, i_{t}}^{\varepsilon_{t}-\eta_{t}}\left(M_{2}\right), \\
\prod_{1 \leq t \leq \ell(\underline{k})} \lambda_{k_{t}, i_{t}}^{\varepsilon_{t}-\eta_{t}}\left(N_{1}\right) \lambda_{k_{t}, i_{t}}^{\eta_{t}-v_{t}}\left(N_{2}\right) \lambda_{k_{t}, i_{t}}^{v_{t}}\left(M_{2}\right),
\end{gathered}
$$

must have the same coefficient. Since $\left(\begin{array}{c}\varepsilon_{t} \\ \eta_{t}\end{array}\right)\left(\begin{array}{l}\eta_{t} \\ v_{t}\end{array}\right)=\left(\begin{array}{c}\varepsilon_{t} \\ \varepsilon_{t}-v_{t}\end{array}\right)\left(\begin{array}{c}\varepsilon_{t}-v_{t} \\ \varepsilon_{t}-\eta_{t}\end{array}\right)$, we have

$$
\alpha_{m, l}^{(\underline{\eta})}=\alpha_{m, l}^{(\varepsilon-\underline{v})}=\alpha_{m, l}^{(\underline{v})}
$$

Now, consider any $\eta$ and $\underline{v}$ with $\underline{0}<\underline{\eta}, \underline{v}<\underline{\varepsilon}$. Either there is $\underline{\tau}>\underline{0}$ with $\underline{\tau} \leq \underline{\eta}$ and $\underline{\tau} \leq \underline{v}$, or we have $\underline{\eta} \leq \underline{\varepsilon}-\underline{v}$. In both cases, we get $\alpha \underline{\eta}_{m, \iota}=\alpha^{\left({ }^{(v)}\right.}{ }_{m, \iota}$. Finally,

$$
\begin{aligned}
\lambda\left(M_{1} \sharp M_{2}\right)=\lambda & \left(M_{1}\right)+\lambda\left(M_{2}\right) \\
& +\sum_{m=1}^{n} \sum_{\iota=(\underline{k}, \underline{i}, \underline{\varepsilon}) \in \mathcal{T}_{m}^{\pi}} \alpha_{m, \iota} \sum_{\underline{0}<\underline{\eta}<\underline{\varepsilon}} \prod_{1 \leq t \leq \ell(\underline{k})}\left(\begin{array}{l}
\varepsilon_{t} \\
\eta_{t}
\end{array}\right) \lambda_{k_{t}, i_{t}}^{\eta_{t}}\left(M_{1}\right) \lambda_{k_{t}, i_{t}}^{\varepsilon_{t}-\eta_{t}}\left(M_{2}\right),
\end{aligned}
$$

where $\alpha_{m, l}$ is the common value of the $\alpha^{(\underline{\eta})}{ }_{m, l}$.

Back to the proof of Proposition 6.9, use the constants $\alpha_{m, \iota}$ given by Lemma 6.10 to define an invariant $\tilde{\lambda}$ :

$$
\tilde{\lambda}=\lambda-\sum_{m=1}^{n} \sum_{\iota=(\underline{k}, \underline{i}, \underline{\varepsilon}) \in \mathcal{T}_{m}^{\pi}} \alpha_{m, \iota} \prod_{1 \leq t \leq \ell(\underline{k})} \lambda_{k_{t}, i_{t}}^{\varepsilon_{t}} .
$$

It is easy to see that $\tilde{\lambda}$ is additive. Thus $\lambda \in \mathcal{I}_{n}^{c} \oplus \mathcal{I}_{n}^{\pi}$, and $\left(\lambda_{k, i}\right)_{0<k \leq n, i \in \mathcal{T}_{k}^{\pi}}$ is a basis of $\mathcal{I}_{n}^{\pi}$. 
It remains to show that $\left(G_{k, i}^{(n)}\right)_{0<k \leq n, i \in \mathcal{T}_{k}}$ is a basis of $\mathcal{F}_{1} / \mathcal{F}_{n+1}$. It suffices to show that $\left(G_{n, i}^{(n)}\right)_{i \in \mathcal{T}_{n}}$ is a basis of $\mathcal{G}_{n}$. Consider $G \in \mathcal{G}_{n}$. We shall prove that the sum $\sum_{i \in \mathcal{T}_{n}} \lambda_{n, i}(G) G_{n, i}^{(n)}$ is finite and equal to $G$ in $\mathcal{G}_{n}$. The term $G$ is a finite linear combination of $\mathbb{Q} H S s$. Let $C_{1}(G) \subset C_{1}$ denote the set of all prime integers $p$ such that $v_{p}(M) \neq 0$ for a $\mathbb{Q H S} M$ in this combination. The set $C_{1}(G)$ is finite. If an invariant $\lambda_{n, i}$ is a multiple of an invariant $v_{p}$ for some $p \notin C_{1}(G)$, then $\lambda_{n, i}(G)=0$. Thus if $\lambda_{n, i}(G) \neq 0$, then $\lambda_{n, i}$ is a product of invariants $v_{p}$ for $p \in C_{1}(G)$ and $\lambda_{k, j}$ for $1<k \leq n$ and $j \in C_{k}$. Recall the set $C_{k}$ is finite for all $k>1$. Hence the sum $\sum_{i \in \mathcal{T}_{n}} \lambda_{n, i}(G) G_{n, i}^{(n)}$ is well defined in $\mathcal{G}_{n}$, and is equal to $G$ since the $\lambda_{n, i}$ generate $\mathcal{I}_{n} / \mathcal{I}_{n-1}$.

Lemma 6.11 Let $M$ and $N$ be $\mathbb{Q H S s}$. For $n>0$,

$$
\begin{aligned}
((M-N) & \left.\in \mathcal{F}_{n+1}\right) \\
& \Leftrightarrow\left(Z_{k, K K T}(M-N)=0 \text { for all } k \leq \frac{1}{2} n \text { and }\left|H_{1}(M ; \mathbb{Z})\right|=\left|H_{1}(N ; \mathbb{Z})\right|\right) .
\end{aligned}
$$

Proof The direct implication is clear since the $Z_{k, K K T}, k \leq n / 2$, and the $v_{p}, p$ prime, are finite type invariants of degree at most $n$. To see that Proposition 6.9 implies the converse implication, recall that the invariants $\lambda_{k, i}$, for $0<k \leq n$ and $i \in \mathcal{T}_{k}$, were defined in Section 6.2 as products of linear combinations of the $v_{p}, p$ prime, and the $\lambda_{k, i}, 0<k \leq n, i \in C_{k}$, that were defined from the $Z_{k, K K T}, k \leq n / 2$ in Lemma 5.16.

Proof of Theorem 1.1 According to Le, Murakami and Ohtsuki [9], $p^{c} \circ Z_{L M O}$ is additive under connected sum, where $p^{c}$ is the projection defined in Lemma 5.16. In [11], Massuyeau proved that $Z_{L M O}$ satisfies the same splitting formulae as $Z_{K K T}$. Thus the invariants $\left(\lambda_{n, i}\right)_{i \in C_{n}}$ of Lemma 5.16 could have been defined with $Z_{L M O}$ instead of $Z_{K K T}$. Therefore, Lemma 6.11 holds for $Z_{L M O}$ instead of $Z_{K K T}$ as well.

\section{References}

[1] E Auclair, C Lescop, Clover calculus for homology 3-spheres via basic algebraic topology, Algebr. Geom. Topol. 5 (2005) 71-106 MR2135546

[2] D Bar-Natan, On the Vassiliev knot invariants, Topology 34 (1995) 423-472 MR1318886

[3] S Garoufalidis, M Goussarov, M Polyak, Calculus of clovers and finite type invariants of 3-manifolds, Geom. Topol. 5 (2001) 75-108 MR1812435

[4] K Habiro, Claspers and finite type invariants of links, Geom. Topol. 4 (2000) 1-83 MR1735632 
[5] A Kawauchi, S Kojima, Algebraic classification of linking pairings on 3-manifolds, Math. Ann. 253 (1980) 29-42 MR594531

[6] M Kontsevich, Vassiliev's knot invariants, from: "I M Gel'fand Seminar", (S Gelfand, S Gindikin, editors), Adv. Soviet Math. 16, Amer. Math. Soc. (1993) 137-150 MR1237836

[7] G Kuperberg, DP Thurston, Perturbative 3-manifold invariants by cut-and-paste topology arXiv:math/9912167

[8] T T Q Le, An invariant of integral homology 3-spheres which is universal for all finite type invariants, from: "Solitons, geometry, and topology: on the crossroad", (V M Buchstaber, S P Novikov, editors), Amer. Math. Soc. Transl. Ser. 2 179, Amer. Math. Soc. (1997) 75-100 MR1437158

[9] T T Q Le, J Murakami, T Ohtsuki, On a universal perturbative invariant of 3manifolds, Topology 37 (1998) 539-574 MR1604883

[10] C Lescop, Splitting formulae for the Kontsevich-Kuperberg-Thurston invariant of rational homology 3-spheres arXiv:math/0411431

[11] G Massuyeau, Splitting formulas for the LMO invariant of rational homology threespheres, in preparation

[12] S V Matveev, Generalized surgeries of three-dimensional manifolds and representations of homology spheres, Mat. Zametki 42 (1987) 268-278, 345 MR915115

[13] M D Meyerson, Representing homology classes of closed orientable surfaces, Proc. Amer. Math. Soc. 61 (1976) 181-182 MR0425967

[14] J W Milnor, J C Moore, On the structure of Hopf algebras, Ann. of Math. 81 (1965) 211-264 MR0174052

[15] R Miranda, Nondegenerate symmetric bilinear forms on finite abelian 2-groups, Trans. Amer. Math. Soc. 284 (1984) 535-542 MR743731

[16] C T C Wall, Quadratic forms on finite groups, and related topics, Topology 2 (1963) 281-298 MR0156890

Institut Fourier

100 rue des maths, $B P$ 74, 38402 St. Martin d'Hères Cedex, France

delphine.moussard@ujf-grenoble.fr

Received: 7 March 2012 Revised: 10 August 2012 